\title{
Embeddings of Danielewski surfaces in affine spaces
}

\author{
A. Dubouloz
}

\begin{abstract}
We construct explicit embeddings of Danielewski surfaces [4] in affine spaces. The equations defining these embeddings are obtained from the $2 \times 2$ minors of a matrix attached to a weighted rooted tree $\gamma$. We characterize those surfaces $S_{\gamma}$ with a trivial Makar-Limanov invariant in terms of their associated trees. We prove that every Danielewski surface $S$ with a nontrivial Makar-Limanov invariant admits a closed embedding in an affine space $\mathbb{A}_{k}^{n}$ in such a way that every $\mathbb{G}_{a, k}$-action on $S$ extends to an action on $\mathbb{A}^{n}$ defined by a triangular derivation. We show that a Danielewski surface $S$ with a trivial Makar-Limanov invariant and non-isomorphic to a hypersurface with equation $x z-P(y)=0$ in $\mathbb{A}_{k}^{3}$ admits nonconjugated algebraically independent $\mathbb{G}_{a, k}$-actions.
\end{abstract}

Mathematics Subject Classification (2000). 14R20,14R25.

Keywords. Danielewski surfaces, additive group actions, Makar-Limanov invariant.

\section{Introduction}

A Danielewski surface over a field $k$ of characteristic zero is an integral affine surface $S$ equipped with a morphism $\pi: S \rightarrow \mathbb{A}_{k}^{1}=\operatorname{Spec}(k[x])$ restricting to the trivial line bundle over $\mathbb{A}_{k}^{1} \backslash\{0\}$ and such that the fiber $\pi^{-1}(0)$ is nonempty and reduced, consisting of a disjoint union of affine lines $\mathbb{A}_{k}^{1}$. For instance, a surface $S_{P, n} \subset$ $\operatorname{Spec}(k[x, y, z])$ with equation $x^{n} z-P(y)=0$, where $P$ is a nonconstant polynomial with $\operatorname{deg}(P)$ simple roots, is a Danielewski surface $\operatorname{pr}_{x}: S_{P, n} \rightarrow \operatorname{Spec}(k[x])$. Danielewski surfaces appear naturally as locally trivial fiber bundles $\rho: S \rightarrow \tilde{X}$ over an affine line with a multiple origin (see e.g. [5]). More precisely, see [4], every such bundle $\rho$ is a principal homogeneous bundle under the action of a line bundle $p: L \rightarrow \tilde{X}$. These principal $L$-bundles are uniquely determined by data consisting of an invertible sheaf $\mathcal{L}$ on $\tilde{X}$ and a Čech 1-cocycle $g$ with values in the dual $\mathcal{L}^{\vee}$ of $\mathcal{L}$ for a suitable covering $U$ of $\tilde{X}$. In turn, the pair $(\mathscr{L}, g)$ is encoded in a combinatorial datum consisting of a rooted tree with weighted edges, which we call a weighted tree (see [4, Example 1.6 and Theorem 3.2] and 2.2 below). Here we use weighted trees in a different way to construct embeddings of Danielewski surfaces into affine spaces. 
More precisely, starting with a suitable class of $k$-weighted trees $\gamma$, we construct explicit ideals of certain polynomial rings. In turn, these ideals define affine surfaces $S_{\gamma}$ which are naturally Danielewski surfaces over the affine line $\mathbb{A}_{k}^{1}$.

The paper is divided as follows. In Section 1 we recall basic facts on weighted trees. We associate to every fine $k$-weighted tree $\gamma=(\Gamma, w)$ (see Definition 1.3 below) a polynomial ring $k[\Gamma]$ and a collection of polynomials in $k[\Gamma]$ defined recursively through the weight function $w$.

In Section 2, we review the construction of Danielewski surfaces as locally trivial bundles over the affine line with an $n$-fold origin given in [4]. Then we associate to every fine $k$-weighted tree $\gamma$ a closed affine subscheme $S_{\gamma}=\operatorname{Spec}\left(B_{\gamma}\right)$ of $\mathbb{A}_{k}^{1} \times$ $\operatorname{Spec}(k[\Gamma])$, and we prove the following result (Theorem 2.9).

Theorem. For every fine $k$-weighted tree $\gamma$, the scheme $S_{\gamma}$ is a Danielewski surface over $\mathbb{A}_{k}^{1}$ for the restriction of the projection $\operatorname{pr}_{1}: \mathbb{A}_{k}^{1} \times \operatorname{Spec}(k[\Gamma]) \rightarrow \mathbb{A}_{k}^{1}$.

For instance, the surface corresponding to the following fine $k$-weighted tree

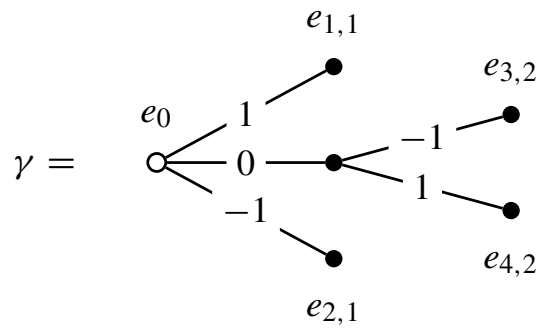

is the Bandman and Makar-Limanov surface [1] $S \subset k[x][y, z, u]$ with equations

$$
x z-y\left(y^{2}-1\right)=0, \quad y u-z\left(z^{2}-1\right)=0, \quad x u-\left(y^{2}-1\right)\left(z^{2}-1\right)=0 .
$$

It is a Danielewski surface over $X=\operatorname{Spec}(k[x])$ via the projection morphism $\mathrm{pr}_{x}: S \rightarrow X$.

Then we show that every embedded Danielewski surface $S_{\gamma}$ as above comes canonically equipped with actions of the additive group $\mathbb{G}_{a, k}$ which are the restrictions to $S_{\gamma}$ of certain $\mathbb{G}_{a, k}$-actions on the ambient space $\mathbb{A}_{k}^{1} \times \operatorname{Spec}(k[\Gamma])$ defined by explicit locally nilpotent derivations $\tilde{\partial}_{\gamma}$ (see Proposition 2.15). In Section 3, we prove the following result (Corollary 3.8).

Theorem. Every Danielewski surface $\pi: S \rightarrow X=\mathbb{A}_{k}^{1}$ is $X$-isomorphic to an embedded Danielewski surface $\pi_{\gamma}: S_{\gamma}=\operatorname{Spec}\left(B_{\gamma}\right) \rightarrow X$ for an appropriate fine $k$-weighted tree $\gamma$.

Moreover, we establish that every $\mathbb{G}_{a, X}$-action on $\pi: S_{\gamma} \rightarrow X$ is induced by a locally nilpotent derivation $\tilde{\partial}_{\gamma}$ as above. As a consequence of this description, we 
deduce that every Danielewski surface $\pi: S \rightarrow X=\mathbb{A}_{k}^{1}$ can be embedded in a relative affine space $\mathbb{A}_{X}^{d}$ in such a way that every $\mathbb{G}_{a, X}$-action on $S$ extends to an action on $\mathbb{A}_{X}^{d}$ (Corollary 3.11). This generalizes a result obtained by Makar-Limanov ([8], [9]) for the Danielewski hypersurfaces $S_{P, n}$ above.

The Makar-Limanov invariant [6] of an affine $k$-scheme $X=\operatorname{Spec}(B)$ is defined as the sub-algebra $\operatorname{ML}(X) \subset B$ consisting of regular functions which are invariant under all $\mathbb{G}_{a, k}$-actions on $X$. If $\operatorname{ML}(X)=k$, then we say that $X$ has a trivial MakarLimanov invariant. For Danielewski surfaces with a nontrivial Makar-Limanov invariant, we prove the following result.

Theorem. Every Danielewski surface with a nontrivial Makar-Limanov invariant can be embedded in an affine space $\mathbb{A}_{k}^{d}=\operatorname{Spec}\left(k\left[x_{1}, \ldots, x_{d}\right]\right)$ in such a way that every $\mathbb{G}_{a, k}$-action on $S$ extends to an action on $\mathbb{A}_{k}^{N}$. Furthermore, every such action is induced by a triangular locally nilpotent derivation of $k\left[x_{1}, \ldots, x_{d}\right]$.

In Section 4, we study Danielewski surfaces with a trivial Makar-Limanov invariant, that is, Danielewski surfaces $S$ which admits two nontrivial $\mathbb{G}_{a, k}$-actions with distinct general orbits. We obtain the following criterion which generalizes Theorem 5.4 in [4].

Theorem. An embedded Danielewski surface $\pi: S_{\gamma}=\operatorname{Spec}\left(B_{\gamma}\right) \rightarrow \mathbb{A}_{k}^{1}$ defined by a fine $k$-weighted tree $\gamma$ has a trivial Makar-Limanov invariant if and only if $\gamma$ is a comb, i.e. a tree such that every element has at most one non-terminal direct descendant (see Definition 4.1 below).

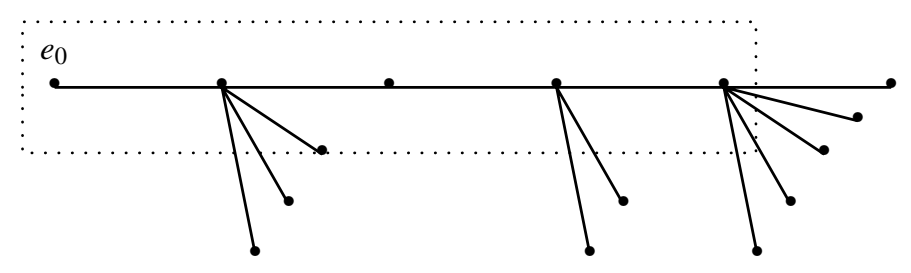

A comb rooted in $e_{0}$.

We obtain the following description (see 4.7 below). For every Danielewski surface $S$ with a trivial Makar-Limanov invariant, there exists a collection of monic polynomials $P_{0}, \ldots, P_{h-1} \in k[t]$ with simple roots $a_{i, j} \in k^{*}, i=0, \ldots, h-1, j=$ $1, \ldots, \operatorname{deg}_{t}\left(P_{i}\right)$, such that $S$ is isomorphic to the nonsingular surface $S_{P_{0}, \ldots, P_{h-1}} \subset$ 
$\operatorname{Spec}\left(k[x]\left[y_{-1}, \ldots, y_{h-2}\right][z]\right)$ defined by the equations

$$
\begin{aligned}
& x z-y_{h-2} \prod_{l=0}^{h-1} P_{l}\left(y_{l-1}\right)=0, \\
& z y_{i-1}-y_{i} y_{h-2} \prod_{l=i+1}^{h-1} P_{l}\left(y_{l-1}\right)=0, \quad x y_{i}-y_{i-1} \prod_{l=0}^{i} P_{l}\left(y_{l-1}\right)=0, \quad 0 \leq i \leq h-2, \\
& y_{i-1} y_{j}-y_{i} y_{j-1} \prod_{l=i+1}^{j} P_{l}\left(y_{l-1}\right)=0, \quad 0 \leq i<j \leq h-2 .
\end{aligned}
$$

On an affine surface $S=\operatorname{Spec}(B)$, two $\mathbb{G}_{a, k}$-actions $\mu_{1}$ and $\mu_{2}$ with associated quotient fibrations $\pi_{1}: S \rightarrow \mathbb{A}_{k}^{1}$ and $\pi_{2}: S \rightarrow \mathbb{A}_{k}^{1}$ respectively are said to be algebraically independent if the general fibers of $\pi_{1}$ and $\pi_{2}$ do not coincide. In this situation, we say that $\mu_{1}$ and $\mu_{2}$ are conjugated if there exists an automorphism $\phi$ of $S$ sending the fibers of $\pi_{1}$ onto the fibers of $\pi_{2}$. This means equivalently that there exists an automorphism $\phi^{*}$ of $B$ such that $\operatorname{Ker}\left(\partial_{2}\right)=\phi^{*}\left(\operatorname{Ker}\left(\partial_{1}\right)\right)$, where $\partial_{1}$ and $\partial_{2}$ denote the locally nilpotent derivations of $B$ corresponding to the actions $\mu_{1}$ and $\mu_{2}$ respectively. Daigle [2] established that all the $\mathbb{G}_{a, k}$-actions on a Danielewski surface $S_{P, 1}=\{x z-P(y)=0\}$ are conjugated. From the explicit description above, we obtain the following result (Theorem 4.12).

Theorem. If a Danielewski surface $S$ non isomorphic to a surface $S_{P, 1}$ admits two independent $\mathbb{G}_{a, k}$-actions, then it admits two algebraically independent nonconjugated $\mathbb{G}_{a, k}$-actions.

We also deduce the following characterization (Corollary 4.13) of the Danielewski surfaces $S_{P, 1}$, which generalizes the ones previously obtained by Bandman and Makar-Limanov [1] and Daigle [2].

Theorem. For a Danielewski surface $\pi: S \rightarrow X=\mathbb{A}_{k}^{1}$ with a trivial Makar-Limanov invariant, the following are equivalent.

1) S admits a free $\mathbb{G}_{a, X}$-action.

2) The canonical sheaf $\omega_{S}$ of $S$ is trivial.

3) $S$ is isomorphic to a surface $S_{P, 1} \subset \mathbb{A}_{k}^{3}=\operatorname{Spec}(k[x, y, z])$ with the equation $x z-P(y)=0$ for a certain nonconstant polynomial $P$ with $\operatorname{deg}(P)$ simple roots.

4) All $\mathbb{G}_{a, k}$-actions on $S$ are conjugated. 


\section{Preliminaries}

Weighted rooted trees. A poset is a nonempty finite partially ordered set $G=(G, \leq)$. A totally ordered subset $C \subset G$ is called a chain of length $l(C)=\operatorname{Card}(C)-1$. $A$ chain which is maximal for the inclusion is called a maximal chain. For every $e \in G$, we let

$$
(\uparrow e)_{G}=\left\{e^{\prime} \in G, e \leq e^{\prime}\right\} \quad \text { and } \quad(\downarrow e)_{G}=\left\{e^{\prime} \in N, e^{\prime} \leq e\right\} .
$$

A subset $\overleftarrow{e^{\prime} e}$ with two elements $e^{\prime}<e$ such that $\left(\uparrow e^{\prime}\right)_{G} \cap(\downarrow e)_{G}=\left\{e^{\prime}<e\right\}$ is called an $e d g e$ of $G$. We denote the set of all edges in $G$ by $E(G)$.

Definition 1.1. A (rooted) tree $\Gamma=(\Gamma, \leq)$ is a poset with a unique minimal element $e_{0}$ called the root, and such that $(\downarrow e)_{\Gamma}$ is a chain for every $e \in \Gamma$. A subposet $\Gamma^{\prime} \subset \Gamma$ which is tree for the induced ordering is called a subtree of $\Gamma$. Given $e \in \Gamma$, the maximal (rooted) subtree of $\Gamma$ rooted in $e$ is the subtree $\Gamma(e)=(\uparrow e)_{\Gamma}$.

1.2. An element $e$ such that $l(\downarrow e)_{\Gamma}=m$ is said to be at level $m$. The maximal elements $e_{i}=e_{i, m_{i}}$, where $m_{i}=l\left(\downarrow e_{i}\right)_{\Gamma}$, of a tree $\Gamma$ are called the leaves of $\Gamma$. We denote the set of those elements by $L(\Gamma)$. The maximal chains of $\Gamma$ are the chains

$$
\Gamma_{e_{i, m_{i}}}=\left(\downarrow e_{i, m_{i}}\right)_{\Gamma}=\left\{e_{i, 0}=e_{0}<e_{i, 1}<\cdots<e_{i, m_{i}}\right\}, \quad e_{i, m_{i}} \in L(\Gamma) .
$$

We say that $\Gamma$ has height $h(\Gamma)=\max \left(m_{i}\right)$. An element of $\Gamma \backslash L(\Gamma)$ is called a parent, and we denote the set of those elements by $\mathbf{P}(\Gamma)$. Given $e \in \Gamma \backslash\left\{e_{0}\right\}$, an element of the chain $\operatorname{Anc}(e)=(\downarrow e) \backslash\{e\}$ is called an ancestor of $e$. The parent of $e$ is the maximal element $\operatorname{Par}(e)$ of $\operatorname{Anc}(e)$. More generally, the $n$-th ancestor of $e$ is defined recursively by $\operatorname{Par}^{n}(e)=\operatorname{Par}\left(\operatorname{Par}^{n-1}(e)\right) \in \operatorname{Anc}(e)$. Given two different elements $e, e^{\prime} \in \Gamma$, the first common ancestor of $e$ and $e^{\prime}$ is the maximal element $\operatorname{Anc}\left(e, e^{\prime}\right)$ of the chain $\operatorname{Anc}(e) \cap \operatorname{Anc}(e)$. If $e$ is not a leaf of $\Gamma$, then the minimal elements of $(\uparrow e)_{\Gamma} \backslash\{e\}$ are called the children of $e$, and we denote the set of those elements by $\mathrm{Ch}(e)$. The degree $\operatorname{deg}(e)$ of an element $e$ is the number of its children.

Definition 1.3. Let $\Gamma$ be a tree. A fine weight function on $\Gamma$, with values in a field $k$, is a function $w: E(\Gamma) \rightarrow k$, which assigns an element $a_{e^{\prime}, e}=w\left(\overleftarrow{e^{\prime} e}\right) \in k$ to every edge $\overleftarrow{e^{\prime} e}$ of $\Gamma$, in such a way that $a_{e^{\prime}, e_{1}} \neq a_{e^{\prime}, e_{2}}$ whenever $e_{1}$ and $e_{2}$ share the same parent $e^{\prime}$. A tree $\Gamma$ equipped with such a function $w$ is referred to as a fine $k$-weighted tree $\gamma=(\Gamma, w)$.

Definition 1.4. An morphism of fine $k$-weighted trees $\tau: \gamma^{\prime}=\left(\Gamma^{\prime}, w^{\prime}\right) \rightarrow \gamma=$ $(\Gamma, w)$ is an order-preserving map $\tau: \Gamma^{\prime} \rightarrow \Gamma$ satisfying the following properties.

a) The image of a maximal subchain of $\Gamma^{\prime}$ is a maximal subchain of $\Gamma$. 
b) For every $e^{\prime} \in \Gamma^{\prime}, \tau^{-1}\left(\tau\left(e^{\prime}\right)\right)$ is either $e^{\prime}$ itself or a maximal subtree of $\Gamma^{\prime}$.

c) For every edge $\overleftarrow{e^{\prime} e}$ of $\Gamma^{\prime}$ such that $\tau(e) \neq \tau\left(e^{\prime}\right)$, we have $w^{\prime}\left(\overleftarrow{e^{\prime} e}\right)=w\left(\overleftarrow{\tau\left(e^{\prime}\right) \tau(e)}\right)$

Remark 1.5. A morphism of fine $k$-weighted trees maps the root $e_{0}^{\prime}$ of $\Gamma^{\prime}$ on the root $e_{0}$ of $\Gamma$ and a leaf $e_{i, m_{i}^{\prime}}^{\prime}$ of $\Gamma^{\prime}$ at level $m_{i}^{\prime}$ onto a leaf $e_{j(i), m_{j(i)}}$ of $\Gamma$ at level $m_{j(i)} \leq m_{i}^{\prime}$. Then b) guarantees that $\tau\left(e_{i, k}^{\prime}\right)=e_{j, \min \left(m_{j(i)}, k\right)}$ for every $k=0, \ldots, m_{i}^{\prime}$, and so, condition c) above makes sense.

Genealogical matrix of a weighted tree. Here we associate to every fine $k$-weighted tree $\gamma=(\Gamma, w)$ a matrix with coefficients in a polynomial ring $k[\Gamma]$.

Definition 1.6. Given a tree $\Gamma$ rooted in $e_{0}$, we associate to every parent $e \in \mathbf{P}(\Gamma)$ a symbol $X_{e}$. If $e^{\prime} \in \mathbf{P}(\Gamma)$ is the parent of a given $e \in \mathbf{P}(\Gamma)$, then we will sometimes denote $X_{e^{\prime}}$ as $X_{\operatorname{Par}(e)}$. We also extend this relationship between the $X_{e}$ 's by introducing the symbol $X_{e_{-1}}=X_{\operatorname{Par}\left(e_{0}\right)}$. We let $k[\Gamma]=k\left[\left(X_{e}\right)_{e \in \mathbf{P}(\Gamma) \cup\left\{e_{-1}\right\}}\right]$ be the corresponding polynomial ring in $d(\Gamma)=\operatorname{Card}(\mathbf{P}(\Gamma))+1$ variables.

For every element $e \in \mathbf{P}(\Gamma)$ of a given fine $k$-weighted tree $\gamma=(\Gamma, w)$ rooted in $e_{0}$, we introduce below three polynomials $F_{e}(\gamma), A_{e}(\gamma), G_{e}(\gamma) \in k[\Gamma]$, defined recursively through the weight function $w: E(\Gamma) \rightarrow k, \overleftarrow{e^{\prime} e} \mapsto a_{e^{\prime}, e}=w\left(\overleftarrow{e^{\prime} e}\right)$

Definition 1.7. For every $e^{\prime} \in \mathbf{P}(\Gamma)$ and every subset $J \subset \mathrm{Ch}\left(e^{\prime}\right)$ we let

$$
F_{e^{\prime}}^{J}=F_{e^{\prime}}^{J}(\gamma)=\prod_{e \in\left(\operatorname{Ch}\left(e^{\prime}\right) \backslash J\right)}\left(X_{\operatorname{Par}\left(e^{\prime}\right)}-a_{e^{\prime}, e}\right) \in k\left[X_{\operatorname{Par}\left(e^{\prime}\right)}\right] \subset k[\Gamma] .
$$

The polynomial $F_{e^{\prime}}:=F_{e^{\prime}}^{\emptyset}$ is called the fatherhood polynomial of $e^{\prime}$.

The ancestral polynomial $A_{e}=A_{e}(\gamma)$ of $e \in \Gamma$ is the polynomial defined recursively by

$$
A_{e_{0}}=1 \quad \text { and } \quad A_{e}=F_{\operatorname{Par}(e)}^{\{e\}} A_{\operatorname{Par}(e)} \in k\left[X_{e_{-1}},\left(X_{e^{\prime}}\right)_{e^{\prime} \in \operatorname{Anc}(\operatorname{Par}(e))}\right] \subset k[\Gamma] .
$$

The genealogical polynomial of $e \in \mathbf{P}(\Gamma)$ with respect to $e^{\prime} \in \operatorname{Anc}(e)$ is the polynomial

$$
G_{e^{\prime}, e}=G_{e^{\prime}, e}(\gamma)=A_{e^{\prime}}^{-1} A_{e} F_{e} \in k\left[X_{e_{-1}},\left(X_{e^{\prime \prime}}\right)_{e^{\prime \prime} \in \operatorname{Anc}(e) \backslash \operatorname{Anc}\left(\operatorname{Par}\left(e^{\prime}\right)\right)}\right] \subset k[\Gamma] .
$$

The polynomial $G_{e}=G_{e_{0}, e}$ is simply referred to as the genealogical polynomial of $e$.

Remark 1.8. Up to changing the variables, $G_{e^{\prime}, e}(\gamma)$ coincides with the genealogical polynomial $G_{e}\left(\gamma^{\prime}\right)$ of $e$ as an element of the maximal weighted subtree $\gamma\left(e^{\prime}\right)=$ $\left(\left(\uparrow e^{\prime}\right)_{\Gamma},\left.w\right|_{\Gamma\left(e^{\prime}\right)}\right)$ of $\gamma$ rooted in $e^{\prime}$, considered as a fine $k$-weighted tree disregarding the inclusion $\gamma\left(e^{\prime}\right) \hookrightarrow \gamma$. 
Definition 1.9. The genealogical matrix of a fine $k$-weighted tree $\gamma=(\Gamma, w)$ is the matrix $M(\gamma) \in \operatorname{Mat}_{d(\Gamma)-1,2}(k[\Gamma])$ with the rows $M_{e}=\left(G_{e}, X_{e}\right) \in \operatorname{Mat}_{1,2}(k[\Gamma])$, $e \in \mathbf{P}(\Gamma)$.

\section{Danielewski surfaces defined by weighted trees}

In [4], the author gives a method to construct a Danielewski surface $\pi: S^{\gamma} \rightarrow X$ over $X=\operatorname{Spec}(k[x])$ from the data consisting of a fine $k$-weighted tree $\gamma$. Here we review briefly this construction. Then we introduce a new procedure to associate to every such tree $\gamma$ a second Danielewski surface $\pi: S_{\gamma} \rightarrow X$, which comes embedded in a relative affine space $\mathbb{A}_{X}^{d}=X \times \mathbb{A}_{k}^{d}$.

Notation 2.1. Throughout this section, we fix a field $k$ of characteristic zero. We let $A=k[x], X=\operatorname{Spec}(A) \simeq \mathbb{A}_{k}^{1}$, and we denote by $X_{*} \simeq \operatorname{Spec}\left(A_{x}\right)$ the open complement in $X$ of the origin $x_{0} \in \mathbb{A}_{k}^{1}$. We consider Danielewski surfaces over the fixed base $X$. We denote by $\operatorname{pr}_{X}: \mathbb{A}_{X}^{1}=\operatorname{Spec}\left(A\left[X_{e_{-1}}\right]\right) \rightarrow X$ the trivial line bundle over $X$. The additive group scheme with base $X$ is denoted by $\mathbb{G}_{a, X}=\operatorname{Spec}(A[T])$.

Abstract Danielewski surface defined by a fine $\boldsymbol{k}$-weighted tree. Given a fine $k$-weighted tree $\gamma=(\Gamma, w)$ of height $h=h(\Gamma)$ with leaves $e_{1, m_{1}}, \ldots, e_{n, m_{n}}$, we construct a Danielewski surface $\pi: S^{\gamma} \rightarrow X$ as follows. Using the maximal weighted subchains

$\gamma_{e_{i, m_{i}}}=\left(\left(\downarrow e_{i, m_{i}}\right), w\right)=\left\{e_{0}=e_{i, 0}<e_{i, 1}<\cdots<e_{i, m_{i}-1}<e_{i, m_{i}}\right\}_{w}, \quad i=1, \ldots, n$,

of $\gamma$, we define a collection of polynomials

$$
\sigma=\left\{\sigma_{i}=\sum_{j=0}^{m_{i}-1} w\left(\overleftarrow{e_{i, j} e_{i, j+1}}\right) x^{j} \in k[x]\right\}_{i=1, \ldots, n}
$$

For every $i \neq j$, we let $g_{i j}=x^{-m_{i}}\left(\sigma_{j}-\sigma_{i}\right) \in A_{x}$. These transition functions $g_{i j}$ satisfy the cocycle relation $g_{i k}=g_{i j}+x^{m_{j}-m_{i}} g_{j k}$ in $A_{x}$ for every triple $i \neq j \neq k$.

2.2. We let $\pi: S^{\gamma} \rightarrow X$ be the $X$-scheme obtained by gluing $n$ copies $S_{i}=$ $\operatorname{Spec}\left(A\left[T_{i}\right]\right)$ of $\mathbb{A}_{X}^{1}$ over $X_{*}$ by means of the $A_{X}$-algebra isomorphisms

$$
\tau_{i j}: A_{x}\left[T_{i}\right] \rightarrow A_{x}\left[T_{j}\right], \quad T_{i} \mapsto g_{i j}+x^{m_{j}-m_{i}} T_{j}, \quad i \neq j, i, j=1, \ldots, n .
$$

Since $\gamma$ is a fine $k$-weighted tree, it follows from 2.8 in [4] that $S^{\gamma}$ is a Danielewski surface $\pi: S^{\gamma} \rightarrow X$. The irreducible components of $\pi^{-1}\left(x_{0}\right)$ are the curves $C_{i}=$ $\pi^{-1}\left(x_{0}\right) \cap S_{i} \simeq \operatorname{Spec}\left(k\left[T_{i}\right]\right), i=1, \ldots, n$. It comes equipped with a canonical birational $X$-morphism $\psi: S^{\gamma} \rightarrow \mathbb{A}_{X}^{1}=\operatorname{Spec}\left(A\left[X_{e_{-1}}\right]\right)$ corresponding to the section $s_{e_{-1}} \in B^{\gamma}=\Gamma\left(S^{\gamma}, \mathcal{O}_{S \gamma}\right)$ with restrictions $\left.s_{e_{-1}}\right|_{S_{i}}=\sigma_{i}+x^{m_{i}} T_{i} \in A\left[T_{i}\right]$, 
$i=1, \ldots, n$. By Theorem 3.2 in [4], every Danielewski surface $\pi: S \rightarrow X$ is $X$-isomorphic to an abstract Danielewski surface $\pi: S^{\gamma} \rightarrow X$ obtained by this procedure.

2.3. A Danielewski surface $\pi: S \rightarrow X$ admits nontrivial actions of the additive group scheme $\mathbb{G}_{a, X}$. Indeed, since by definition $\left.S\right|_{X_{*}}$ is isomorphic to the trivial line bundle $\mathbb{A}_{X_{*}}^{1}=\operatorname{Spec}\left(A_{x}\left[X_{e_{-1}}\right]\right)$ over $X_{*}$, there exists $r \geq 0$ such that the $A$-derivation $x^{m} \partial_{X_{e_{-1}}}$ extends to a locally nilpotent $A$-derivation $\partial$ of $\Gamma\left(S, \mathcal{O}_{S}\right)$, corresponding to a nontrivial $\mathbb{G}_{a, X}$-action on $S$. By Proposition 2.12 in [4], every nontrivial $\mathbb{G}_{a, X}$-action on a Danielewski surface $S^{\gamma}$ is induced by the extension $\partial_{a, m}$ to $B^{\gamma}$ of a locally nilpotent $A$-derivation $a x^{m} \partial_{X_{e_{-1}}}$ of $B^{\gamma} \otimes_{A} A_{x} \simeq A_{x}\left[X_{e_{-1}}\right]$, where $m \geq h(\Gamma)$ and $a \in A \backslash\{0\}$. We denote the corresponding $\mathbb{G}_{a, X}$-actions on $\mathbb{A}_{X}^{1}$ and $S^{\gamma}$ by $\boldsymbol{t}_{a, m}$ and $\boldsymbol{t}_{a, m}^{\gamma}$ respectively. On the open subsets $S_{i}=\operatorname{Spec}\left(A\left[T_{i}\right]\right), \mathfrak{t}_{a, m}^{\gamma}$ coincides with the twisted translation $\boldsymbol{t}_{a, m-m_{i}}$ defined by the group co-action homomorphism

$$
A\left[T_{i}\right] \rightarrow A\left[T_{i}, T\right] \simeq A\left[T_{i}\right] \otimes_{A} A[T], \quad T_{i} \mapsto T_{i}+a x^{m-m_{i}} T, \quad i=1, \ldots, n .
$$

The canonical morphism $\psi: S^{\gamma} \rightarrow \mathbb{A}_{X}^{1}$ is $\mathbb{G}_{a, X}$-equivariant when $S^{\gamma}$ and $\mathbb{A}_{X}^{1}$ are equipped with the $\mathbb{G}_{a, X}$-actions $\boldsymbol{t}_{a, m}^{\gamma}$ and $\boldsymbol{t}_{a, m}$ respectively.

Example 2.4. The collection of polynomials $\sigma$ corresponding to the following fine $k$-weighted tree $\gamma=(\Gamma, w)$ with leaves $e_{1,1}, e_{2,1}, e_{3,2}, e_{4,2}$

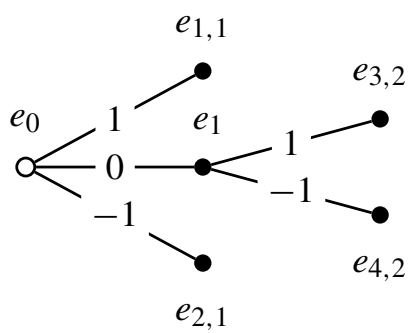

is $\sigma=\{1,-1, x,-x\}$. The associated transition functions $g=\left\{g_{i j}\right\}_{1 \leq i<j \leq 4}$ are

$$
\begin{gathered}
g_{12}=g_{34}=-2 x^{-1}, \quad g_{13}=-g_{24}=x^{-1}(x-1), \\
g_{23}=-g_{14}=x^{-1}(x+1) .
\end{gathered}
$$

The gluing homomorphisms $\left\{\tau_{i j}\right\}_{1 \leq i<j \leq 4}$ are given by

$$
\begin{aligned}
\tau_{i j}: k\left[x, x^{-1}\right]\left[T_{i}\right] & \rightarrow k\left[x, x^{-1}\right]\left[T_{j}\right], \\
T_{i} & \mapsto \begin{cases}g_{i j}+T_{j}, & \text { if }(i, j) \in\{(1,2),(3,4)\}, \\
g_{i j}+x T_{j}, & \text { if }(i, j) \in\{(1,3),(1,4),(2,3),(2,4)\} .\end{cases}
\end{aligned}
$$


The $\mathbb{G}_{a, X}$-action $\boldsymbol{t}_{1,2}^{\gamma}$ on $\pi: S^{\gamma} \rightarrow X$ is a non-free action which restricts on $S_{i}=$ $\operatorname{Spec}\left(A\left[T_{i}\right]\right)$ to the action

$$
T_{i} \mapsto \begin{cases}T_{i}+x T, & \text { if } i=1,2, \\ T_{i}+T, & \text { if } i=3,4 .\end{cases}
$$

Letting $P(t)=t^{2}-1 \in k[t]$, we will see in Example 3.2 below that $S^{\gamma}$ is $X$ isomorphic to the Bandman and Makar-Limanov surface [1] $S \subset \operatorname{Spec}(k[x][y, z, u])$ with equations

$$
x z-y P(y)=0, \quad y u-z P(z)=0, \quad x u-P(y) P(z)=0,
$$

and that $\boldsymbol{t}_{1,2}^{\gamma}$ coincides with the action on $S$ induced by the triangular derivation $\partial_{1,2}=x^{2} \partial_{y}+x\left(3 y^{2}-1\right) \partial_{z}+\left(2 P(y)\left(3 y^{2}-1\right) z+2 x y P(z)\right) \partial_{u} \in \operatorname{Der}_{k[x]}(k[x][y, z, u])$.

Embedded Danielewski surface defined by a fine $\boldsymbol{k}$-weighted tree. Given a fine $k$-weighted tree $\gamma=(\Gamma, w)$, we construct a Danielewski surface $\pi: S_{\gamma} \rightarrow X$ which comes embedded in a relative affine space $\mathbb{A}_{X}^{d(\Gamma)}$, where $d(\Gamma)=\operatorname{Card}(\mathbf{P}(\Gamma))+1$. These surfaces are canonically equipped with the restrictions of certain actions of the additive group $\mathbb{G}_{a, X}$ on the ambient space $\mathbb{A}_{X}^{d(\Gamma)}$, defined by explicit locally nilpotent derivations.

2.5. Given a fine $k$-weighted tree $\gamma=(\Gamma, w)$, we let $A[\Gamma]=A \otimes_{k} k[\Gamma] \simeq$ $A\left[X_{e_{-1}},\left(X_{e}\right)_{e \in \mathbf{P}(\Gamma)}\right]$ (see Definition 1.6). We let $\bar{M}(\gamma) \in \operatorname{Mat}_{d(\Gamma), 2}(A[\Gamma])$ be the matrix with the rows $M_{e_{-1}}=(x, 1)$ and $M_{e}=\left(G_{e}(\gamma), X_{e}\right), e \in \mathbf{P}(\Gamma)$, i.e. $\bar{M}(\gamma)=\left(M_{e_{-1}}, M(\gamma)\right)$, where $M(\gamma) \in \operatorname{Mat}_{d(\Gamma)-1,2}(k[\Gamma])$ denotes the genealogical matrix of $\gamma$ (Definition 1.9).

Definition 2.6. Given a fine $k$-weighted tree $\gamma=(\Gamma, w)$, we let $I_{\gamma} \subset A[\Gamma]$ be the ideal generated by the simplified genealogical minors of $\bar{M}(\gamma)$

$$
\Delta_{e^{\prime}, e}=\Delta_{e^{\prime}, e}(\gamma)=A_{e^{\prime}}^{-1} \operatorname{det}\left(M_{\operatorname{Par}\left(e^{\prime}\right)}, M_{e}\right) \in A[\Gamma], \quad\left(e, e^{\prime}\right) \in \mathbf{P}(\Gamma) \times(\downarrow e)_{\Gamma} .
$$

We let $B_{\gamma}=A[\Gamma] / I_{\gamma}$, and we let $\pi: S_{\gamma}=\operatorname{Spec}\left(B_{\gamma}\right) \rightarrow X$ be the corresponding closed sub- $X$-scheme of the relative affine space $\mathbb{A}_{X}^{d(\Gamma)}=\operatorname{Spec}(A[\Gamma])$.

2.7. By construction, $\Delta_{e}:=\Delta_{e_{0}, e}=x X_{e}-G_{e} \in A\left[\left(X_{e^{\prime}}\right)_{e^{\prime} \in(\downarrow e)_{\Gamma} \cup\left\{e_{-1}\right\}}\right]$ for every $e \in \mathbf{P}(\Gamma)$, whereas $\Delta_{e^{\prime}, e}=\left(X_{\operatorname{Par}^{2}\left(e^{\prime}\right)}-a_{\operatorname{Par}\left(e^{\prime}\right), e^{\prime}}\right) X_{e}-X_{\operatorname{Par}\left(e^{\prime}\right)} G_{e^{\prime}, e}$ for every pair $\left(e, e^{\prime}\right) \in \mathbf{P}(\Gamma) \times\left((\downarrow e)_{\Gamma} \backslash\left\{e_{0}\right\}\right)$. As a consequence, for every triple $e_{0}<e^{\prime \prime} \leq e^{\prime} \leq e$ in $\mathbf{P}(\Gamma)$, the following relations hold in $A[\Gamma]$ :

$$
\left.\begin{array}{rl}
A_{e^{\prime}} \Delta_{e^{\prime}, e} & =X_{\operatorname{Par}\left(e^{\prime}\right)} \Delta_{e}-\Delta_{\operatorname{Par}\left(e^{\prime}\right)} X_{e}, \\
x \Delta_{e^{\prime}, e} & =\left(X_{\operatorname{Par}^{2}\left(e^{\prime}\right)}-a_{\operatorname{Par}\left(e^{\prime}\right), e^{\prime}}\right) \Delta_{e}-\Delta_{\operatorname{Par}\left(e^{\prime}\right)} G_{e^{\prime}, e}, \\
\left(X_{\operatorname{Par}^{2}\left(e^{\prime \prime}\right)}-a_{\operatorname{Par}\left(e^{\prime \prime}\right), e^{\prime \prime}}\right) \Delta_{e^{\prime}, e} & =\left(X_{\operatorname{Par}^{2}\left(e^{\prime}\right)}-a_{\operatorname{Par}\left(e^{\prime}\right), e^{\prime}}\right) \Delta_{e^{\prime \prime}, e}-\Delta_{e^{\prime \prime}, e^{\prime}} G_{e^{\prime}, e} .
\end{array}\right\}
$$


2.8. If $\gamma=(\Gamma, w)$ is the trivial tree with just one element $e_{0}$, then the first projection $\pi: S_{\gamma}=\operatorname{Spec}\left(k[x]\left[X_{e_{-1}}\right]\right) \rightarrow X$ is a Danielewski surface. Similarly, if $\Gamma$ has height 1 , then $G_{e_{0}} \in k\left[X_{e_{-1}}\right]$ is a monic polynomial with simple roots $a_{e_{0}, e}=$ $w\left(\overleftarrow{e_{0} e}\right) \in k, e \in \mathrm{Ch}\left(e_{0}\right)$. Therefore,

$$
\pi: S_{\gamma}=\operatorname{Spec}\left(A[\Gamma] / I_{\gamma}\right)=\operatorname{Spec}\left(k[x]\left[X_{e_{-1}}, X_{e_{0}}\right] / x X_{e_{0}}-G_{e_{0}}\left(X_{e_{-1}}\right)\right) \rightarrow X
$$

is a Danielewski surface, and the irreducible components of $\pi^{-1}\left(x_{0}\right)$ are the curves $C_{e} \simeq \operatorname{Spec}\left(k\left[X_{e_{0}}\right]\right)$ with defining ideals $I_{\gamma, e}=\left(I_{\gamma}, X_{e_{-1}}-a_{e, e_{0}}\right) \subset A[\Gamma], e \in$ $\mathrm{Ch}\left(e_{0}\right)$. More generally, we have the following result.

Theorem 2.9. For every fine $k$-weighted tree $\gamma=(\Gamma, w)$ with leaves $e_{1, m_{1}}, \ldots, e_{n, m_{n}}$, $\pi: S_{\gamma} \rightarrow X$ is a Danielewski surface. Furthermore, the fiber $\pi^{-1}\left(x_{0}\right)$ is the disjoint union of the curves $C_{e_{i, m_{i}}} \simeq \operatorname{Spec}\left(k\left[X_{e_{i, m_{i}-1}}\right]\right)$ with defining ideals

$$
I_{\gamma, e_{i, m_{i}}}=\left(I_{\gamma}, x,\left(X_{e_{i, j-1}}-a_{e_{i, j}, e_{i, j+1}}\right)_{0 \leq j \leq m_{i}-1}\right) \subset A[\Gamma], \quad i=1, \ldots, n .
$$

The proof is divided as follows. In 2.10, Lemmas 2.11 and 2.12 below, we show that $S_{\gamma}$ is an integral scheme. Then, in Lemma 2.13, we describe explicitly the irreducible components of $\pi^{-1}\left(x_{0}\right)$.

2.10. We first observe that $S_{\gamma}$ restricts to the trivial line bundle $\mathbb{A}_{X_{*}}^{1}=\operatorname{Spec}\left(A_{x}\left[X_{e_{-1}}\right]\right)$ over $X_{*}$. Indeed, the second relation of (2.2) guarantees that the ideal $I_{\gamma} A_{x}[\Gamma]$ of $A_{x}[\Gamma] \simeq A[\Gamma] \otimes_{A} A_{x}$ is generated by the polynomials $x^{-1} \Delta_{e}=X_{e}-x^{-1} G_{e}$, $e \in \mathbf{P}(\Gamma)$. Since $G_{e}$ only involves the variables $X_{e^{\prime}}$, where $e^{\prime} \in \operatorname{Anc}(e)$, we recursively arrive at an $A_{x}$-algebra isomorphism $A_{x}[\Gamma] / I_{\gamma} A_{x}[\Gamma] \simeq A_{x}\left[X_{e_{-1}}\right]$. Thus $S_{\gamma}$ is a Danielewski surface with base $(k[x], x)$ provided that $x$ is not a zero divisor in $B_{\gamma}$ and that $B_{\gamma} / x B_{\gamma}$ is isomorphic to a nonempty direct product of polynomial rings in one variable over $k$. Indeed, the first condition guarantees that the canonical map $B_{\gamma} \rightarrow B_{\gamma} \otimes_{A} A_{x} \simeq A_{x}\left[X_{e_{-1}}\right]$ is injective. In turn, this implies that $B_{\gamma}$ is a sub-domain of $A_{x}\left[X_{e_{-1}}\right]$. The second one means equivalently that the fiber $\pi^{-1}\left(x_{0}\right)$ decomposes as a nonempty disjoint union of affine lines $\mathbb{A}_{k}^{1}$.

To show that $x$ is not a zero divisor in $B_{\gamma}$, it suffices to find a covering of $S_{\gamma}$ by principal affine open subsets $Y_{i}=\operatorname{Spec}\left(B_{i}\right)$ such that $x$ is not a zero divisor in $B_{i}$ for every $i=1, \ldots, n$.

Lemma 2.11. If $\gamma=(\Gamma, w)$ if a fine $k$-weighted tree with the leaves $e_{1}, \ldots, e_{n}$, then $S_{\gamma}$ is covered by the principal open subsets $Y_{i}=\operatorname{Spec}\left(A[\Gamma][T] /\left(I_{\gamma}, A_{e_{i}} T-1\right)\right)$, $i=1, \ldots, n$.

Proof. For every $e \in \mathbf{P}(\Gamma)$ the polynomials $F_{e}^{\left\{e^{\prime}\right\}} \in A\left[X_{\operatorname{Par}(e)}\right], e^{\prime} \in \mathrm{Ch}(e)$ generate the unit ideal of $A\left[X_{\operatorname{Par}(e)}\right]$ as $\gamma$ is a fine $k$-weighted tree. Therefore, there exist 
polynomials $\Lambda_{e^{\prime}} \in A[\Gamma], e^{\prime} \in \mathrm{Ch}(e)$, such that

$$
A_{e}=A_{e} \sum_{e^{\prime} \in \operatorname{Ch}(e)} \Lambda_{e^{\prime}} F_{e}^{\left\{e^{\prime}\right\}}=\sum_{e^{\prime} \in \operatorname{Ch}(e)} \Lambda_{e^{\prime}} A_{e^{\prime}} .
$$

It follows by induction that the image of $A_{e_{0}}=1$ in $B_{\gamma}$ belongs to the ideal generated by the images $a_{i} \in B_{\gamma}$ of the ancestral polynomials $A_{e_{i}}$ of the leaves of $\Gamma$. This means equivalently that the open subsets $\operatorname{Spec}\left(\left(B_{\gamma}\right)_{a_{i}}\right) \simeq \operatorname{Spec}\left(A[\Gamma][T] /\left(I_{\gamma}, A_{e_{i}} T-1\right)\right)$ cover $S_{\gamma}$.

Lemma 2.12. For every $i=1, \ldots, n, Y_{i}$ is an integral scheme.

Proof. Let us denote by $e_{j}=e_{i, j}, j=0, \ldots, m=m_{i}$, the elements of the maximal subchain $\left(\downarrow e_{i, m_{i}}\right)_{\Gamma}$ of $\Gamma$ associated with the leaf $e_{i, m_{i}}$. For every $i=1, \ldots, m-2$, the polynomial $A_{e_{i+1}}$ divides $A_{e_{m}}$. Similarly, for every $e \in \mathbf{P}(\Gamma) \backslash\left(\downarrow e_{m}\right)$, the first common ancestor of $e$ and $e_{m}$ is an element $e_{i}, i \leq m-1$, such that $e^{\prime}=\operatorname{Ch}\left(e_{i}\right) \cap$ $(\downarrow e) \neq e_{i+1}$, and so $\left(X_{e_{i-1}}-a_{e_{i}, e^{\prime}}\right)$ divides $A_{e_{m}}$. Therefore, these polynomials become invertible in $A[\Gamma]_{A_{e_{m}}}$. We claim that the ideal $I_{\gamma} A[\Gamma]_{A_{e_{m}}}$ is generated by the polynomials

$$
\begin{array}{rlrl}
\delta_{e_{i}} & =A_{e_{i+1}}^{-1} \Delta_{e_{i}}=-\left(X_{e_{i-1}}-a_{e_{i}, e_{i+1}}\right)+A_{e_{i+1}}^{-1} x X_{e_{i}}, & i=1, \ldots, m-2, \\
\delta_{e_{i}, e} & =\left(X_{e_{i-1}}-a_{e_{i}, e^{\prime}}\right)^{-1} \Delta_{e_{i}, e} \\
& =X_{e}-\left(X_{e_{i-1}}-a_{e_{i}, e^{\prime}}\right)^{-1} X_{e_{i}} G_{e_{i}, e}, & & \left\{\begin{array}{l}
e \in \mathbf{P}(\Gamma) \backslash\left(\downarrow e_{m}\right), \\
\operatorname{Anc}\left(e, e_{m}\right)=e_{i}, \\
e^{\prime}=\operatorname{Ch}\left(e_{i}\right) \cap(\downarrow e)_{\Gamma} .
\end{array}\right.
\end{array}
$$

Indeed, the second relation of (2.2) guarantees that the polynomials $\Delta_{e}$, where $e \in$ $\mathbf{P}(\Gamma) \backslash\left(\downarrow e_{m}\right)_{\Gamma}$, can be expressed in $A[\Gamma]_{A_{e_{m}}}$ in terms of the $\delta_{e_{i}}$ 's and $\delta_{e_{i}, e}$ 's. In turn, we deduce from the first and the third ones that all the polynomials $\Delta_{e^{\prime}, e}$, $\left(e, e^{\prime}\right) \in \mathbf{P}(\Gamma) \times\left((\downarrow e)_{\Gamma} \backslash\left\{e_{0}\right\}\right)$ belong to the ideal of $A[\Gamma]_{A_{e_{m}}}$ generated by the $\delta_{e_{i}}$ 's and the $\delta_{e_{i}, e}$ 's. Since the polynomials $A_{e_{i}}$ and $G_{e_{i}, e}$ above only involve the variables corresponding to the elements in $\left(\downarrow e_{i-2}\right)_{\Gamma}$ and $\left(\uparrow e^{\prime}\right)_{\Gamma} \cap(\downarrow \operatorname{Anc}(e))_{\Gamma}$ respectively, we conclude by induction that there exists a nonconstant polynomial $P \in A\left[X_{e_{m-1}}\right]$ such that $A[\Gamma]_{A_{e_{m}}} / I_{\gamma} A[\Gamma]_{A_{e_{m}}} \simeq A\left[X_{e_{m-1}}\right]_{P}$. Since $A$ is a domain and $P$ is nonconstant, it follows that $\left(B_{\gamma}\right)_{a_{i}} \simeq A\left[X_{e_{m-1}}\right]_{P}$ is a nonzero domain too.

Summing up, we have established that for every fine $k$-weighted tree $\gamma$, $\pi: S_{\gamma} \rightarrow X$ is an integral affine scheme restricting to the trivial bundle $\mathbb{A}_{X_{*}}^{1}$ over $X_{*}$. The following result completes the proof of Theorem 2.9.

Lemma 2.13. For every fine $k$-weighted tree $\gamma=(\Gamma, w)$ with leaves $e_{1, m_{1}}, \ldots, e_{n, m_{n}}$, the fiber $\pi^{-1}\left(x_{0}\right)$ of $\pi: S_{\gamma} \rightarrow X$ is the disjoint union of the curves $C_{e_{i, m_{i}}} \simeq$ $\operatorname{Spec}\left(k\left[X_{e_{i, m_{i}-1}}\right]\right)$ with defining ideals

$$
I_{\gamma, e_{i, m_{i}}}=\left(I_{\gamma}, x,\left(X_{e_{i, j-1}}-a_{e_{i, j}, e_{i, j+1}}\right)_{0 \leq j \leq m_{i}-1}\right) \subset A[\Gamma], \quad i=1, \ldots, n .
$$


Proof. We proceed by induction on the height $h$ of $\Gamma$. If $h=0$ then $S_{\gamma}=$ $\operatorname{Spec}\left(A\left[X_{e_{-1}}\right]\right)$ and $\pi^{-1}\left(x_{0}\right) \simeq \operatorname{Spec}\left(k\left[X_{e_{-1}}\right]\right)$. Otherwise, if $\operatorname{Ch}\left(e_{0}\right) \neq \emptyset$ then, since $\gamma$ is a fine $k$-weighted tree, it follows that the polynomials $X_{e_{-1}}-a_{e_{0}, e}, e \in \mathrm{Ch}\left(e_{0}\right)$ are pairwise relatively prime. Therefore $\pi^{-1}\left(x_{0}\right)=\operatorname{Spec}\left(A[\Gamma] /\left(x, I_{\gamma}\right)\right)$ decomposes as the disjoint union of curves $D_{e}=\operatorname{Spec}\left(A[\Gamma] /\left(x, X_{e_{-1}}-a_{e_{0}, e}, I_{\gamma}\right)\right), e \in \operatorname{Ch}\left(e_{0}\right)$. We let $\gamma(e)=\left(\Gamma(e),\left.w\right|_{\Gamma(e)}\right)$ be the maximal fine $k$-weighted subtree of $\gamma$ rooted in $e$. Clearly, the ideal $\left(x, X_{e_{-1}}-a_{e_{0}, e}, I_{\gamma}\right)$ coincides with the ideal $I_{e} \subset A[\Gamma]$ generated by $x, X_{e_{-1}}-a_{e_{0}, e}$ and the polynomials

$$
\begin{array}{ll}
G_{e, e^{\prime}}(\gamma), & e^{\prime} \in \mathbf{P}(\Gamma(e)), \\
\Delta_{e^{\prime \prime}, e^{\prime}}(\gamma), & \left(e^{\prime}, e^{\prime \prime}\right) \in \mathbf{P}(\Gamma(e)) \times\left(\operatorname{Anc}_{\Gamma(e)}\left(e^{\prime}\right)\right), \\
\delta_{e, e^{\prime}}=\left(a_{e_{0}, e}-a_{e_{0}, e^{\prime \prime}}\right) X_{e^{\prime}}-X_{e_{0}} G_{e, e^{\prime}}(\gamma), & \left\{\begin{array}{l}
e^{\prime} \in \mathbf{P}(\Gamma) \backslash\left(\left\{e_{0}\right\} \cup \mathbf{P}(\Gamma(e))\right), \\
e^{\prime \prime}=\operatorname{Ch}\left(e_{0}\right) \cap\left(\downarrow e^{\prime}\right) \neq e .
\end{array}\right.
\end{array}
$$

By definition, we have $A[\Gamma(e)]=A\left[X_{e_{-1}},\left(X_{e^{\prime}}\right)_{e^{\prime} \in \mathbf{P}(\Gamma)}\right] \simeq A\left[X_{e_{0}},\left(X_{e^{\prime}}\right)_{e^{\prime} \in \mathbf{P}(\Gamma)}\right]$ as $e_{0} \notin \Gamma(e)$. This choice of coordinates yields the identities

$$
\begin{aligned}
G_{e^{\prime}}(\gamma(e)) & =G_{e, e^{\prime}}(\gamma), & & e^{\prime} \in \mathbf{P}(\Gamma(e)), \\
G_{e^{\prime \prime}, e^{\prime}}(\gamma(e)) & =G_{e^{\prime \prime}, e^{\prime}}(\gamma), & & \left(e^{\prime}, e^{\prime \prime}\right) \in \mathbf{P}(\Gamma(e)) \times \operatorname{Anc}_{\Gamma(e)}\left(e^{\prime}\right),
\end{aligned}
$$

and we conclude that $A[\Gamma] /\left(x, X_{e_{-1}}-a_{e_{0}, e}, I_{\gamma}\right) \simeq A[\Gamma] / I_{e} \simeq A[\Gamma(e)] /\left(x, I_{\gamma(e)}\right)$. This means equivalently that $\pi^{-1}\left(x_{0}\right)$ is isomorphic to the disjoint union of the fibers $\pi_{\gamma(e)}^{-1}\left(x_{0}\right)$ of the corresponding surfaces $\pi_{\gamma(e)}: S_{\gamma(e)} \rightarrow X, e \in \mathrm{Ch}\left(e_{0}\right)$. Since the fine $k$-weighted tree $\gamma(e)$ has height $h-1$, it follows from our induction hypothesis that these fibers are nonempty and reduced, consisting of disjoint unions of affine lines $\mathbb{A}_{k}^{1}$. So the same holds for $\pi^{-1}\left(x_{0}\right)$. Finally, the precise description of the irreducible components of $\pi^{-1}\left(x_{0}\right)$ follows easily by induction again.

Remark 2.14. A Danielewski surface $\pi: S_{\gamma} \rightarrow X=\mathbb{A}_{k}^{1}$ is a flat (or rather a smooth) $X$-scheme. In general, the scheme $\tilde{\pi}: \tilde{S}_{\gamma} \rightarrow X$ with defining ideal $\tilde{I}_{\gamma}$ generated only by the polynomials $\Delta_{e}, e \in \mathbf{P}(\Gamma)$, is not flat over $X$. The above discussion together with the second relation of (2.2) imply that $S_{\gamma}$ coincides with the flat limit over $X$ of the trivial family of affine lines $\left.\tilde{S}_{\gamma}\right|_{X_{*}} \simeq \mathbb{A}_{X_{*}}^{1}$ defined by the equations $\Delta_{e}=0$, $e \in \mathbf{P}(\Gamma)$, in $\mathbb{A}_{X_{*}}^{d(\Gamma)}=\operatorname{Spec}\left(A_{x}[\Gamma]\right)$. This explains why the polynomials $\Delta_{e^{\prime}, e}$, $\left(e, e^{\prime}\right) \in \mathbf{P}(\Gamma) \times\left((\downarrow e)_{\Gamma} \backslash\left\{e_{0}\right\}\right)$, should be added to the obvious ones $\Delta_{e}, e \in \mathbf{P}(\Gamma)$, to define the surface $S_{\gamma}$.

The following result shows that the embedded Danielewski surface $\pi: S_{\gamma} \rightarrow X$ defined by a fine $k$-weighted tree $\gamma=(\Gamma, w)$ admits nontrivial actions of the additive group $\mathbb{G}_{a, X}$, which come as the restrictions of certain $\mathbb{G}_{a, X}$-actions on the ambient space $\mathbb{A}_{X}^{d(\Gamma)}$. 
Proposition 2.15. Let $\gamma=(\Gamma, w)$ be a fine $k$-weighted tree of height $h \geq 0$. Then, for every $m \geq h$ and every $a \in A \backslash\{0\}$, the derivation $\tilde{\partial}_{\gamma, a, m} \in \operatorname{Der}_{A}\left(A[\Gamma], A_{x}[\Gamma]\right)$ defined recursively by

$$
\tilde{\partial}_{\gamma, a, m}=a x^{m} \partial_{X_{e_{-1}}}+x^{-1} \sum_{e \in \mathbf{P}(\Gamma)} \tilde{\partial}_{\gamma, a, m}\left(G_{e}(\gamma)\right) \partial_{X_{e}}
$$

is a triangular derivation of $A[\Gamma]$ inducing a locally nilpotent A-derivation $\partial_{\gamma, a, m}$ of $B_{\gamma}$.

Proof. It suffices to prove the assertion for the derivation $\tilde{\partial}=\partial_{\gamma, 1, h}$ as $\tilde{\partial}_{\gamma, a, m}=$ $a x^{m-h} \tilde{\partial}$. For every $e \in \mathbf{P}(\Gamma)$ at level $i<h$, the polynomial $G_{e}$ only involves the variables $X_{0}$ and $X_{e^{\prime}}, e^{\prime} \in \operatorname{Anc}(e)$. So we conclude recursively that $\tilde{\partial}\left(X_{e}\right) \in$ $x^{h-i-1} A\left[X_{e_{-1}},\left(X_{e^{\prime}}\right)_{e^{\prime} \in \operatorname{Anc}(e)}\right]$. Thus $\tilde{\partial}$ restricts to a triangular $A$-derivation of $A[\Gamma]$. By construction, $\tilde{\partial}$ annihilates $\Delta_{e}$ for every $e \in \mathbf{P}(\Gamma)$. Moreover, $x \tilde{\partial}\left(\Delta_{e^{\prime}, e}\right)=$ $\tilde{\partial}\left(x \Delta_{e^{\prime}, e}\right) \in I_{\gamma}$ for every pair $\left(e, e^{\prime}\right) \in\left(\mathbf{P}(\Gamma) \backslash\left\{e_{0}\right\}\right) \times\left((\downarrow e)_{\Gamma} \backslash\left\{e_{0}\right\}\right)$ by virtue of (2.2). Thus $\tilde{\partial}\left(\Delta_{e^{\prime}, e}\right) \in I_{\gamma}$ as $I_{\gamma}$ is a prime ideal which does not contain $x$. Hence $\tilde{\partial}\left(I_{\gamma}\right) \subset I_{\gamma}$ and so, $\tilde{\partial}$ induces a locally nilpotent $A$-derivation $\partial$ of $B_{\gamma}$.

Example 2.16. We consider the following fine $k$-weighted tree $\tilde{\gamma}=(\Gamma, \tilde{w})$ with the leaves $e_{1,1}, e_{2,1}, e_{3,2}, e_{4,2}$.

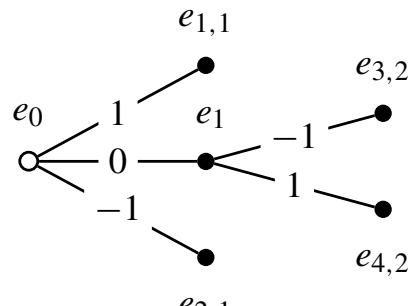

We have $A[\Gamma]=k[x]\left[X_{e_{-1}}, X_{e_{0}}, X_{e_{1}}\right]$ and

$$
{ }^{t} \bar{M}(\tilde{\gamma})=\left(\begin{array}{ccc}
x & X_{e_{-1}} P\left(X_{e_{-1}}\right) & P\left(X_{e_{-1}}\right) P\left(X_{e_{0}}\right) \\
1 & X_{e_{0}} & X_{e_{1}}
\end{array}\right),
$$

where $P(t)=t^{2}-1 \in k[t]$. Therefore $\pi: S_{\tilde{\gamma}} \rightarrow X$ is the surface with equations

$$
\begin{gathered}
x X_{e_{0}}-X_{e_{-1}} P\left(X_{e_{-1}}\right)=0, \quad X_{e_{-1}} X_{e_{1}}-X_{e_{0}} P\left(X_{e_{0}}\right)=0, \\
x X_{e_{1}}-P\left(X_{e_{-1}}\right) P\left(X_{e_{0}}\right)=0 .
\end{gathered}
$$

Letting $y=X_{e_{-1}}, z=X_{e_{0}}$ and $u=X_{e_{1}}$, the locally nilpotent derivation $\tilde{\partial}_{\tilde{\gamma}, 1,2} \in$ $\operatorname{Der}_{A}(A[\Gamma])$ is simply the derivation $\partial_{1,2} \in \operatorname{Der}_{k[x]}(k[x][y, z, u])$ of Example 2.4. 


\section{Embeddings of Danielewski surfaces in affine spaces}

In this section, we compare the two constructions of Danielewski surfaces by means of fine $k$-weighted trees. We describe a certain class of morphisms of Danielewski surfaces as the restrictions of suitable linear projections.

From abstract to embedded Danielewski surfaces. Here we prove the following result.

Theorem 3.1. For every abstract Danielewski surface $\pi: S^{\gamma} \rightarrow X$ defined by a fine $k$-weighted tree $\gamma=(\Gamma, w)$, there exists another fine weight function $\tilde{w}: E(\Gamma) \rightarrow k$ on the tree $\Gamma$, and a closed embedding $\zeta: S^{\gamma} \hookrightarrow \mathbb{A}_{X}^{d(\Gamma)}$ inducing an isomorphism between $S^{\gamma}$ and the embedded Danielewski surface $S_{\tilde{\gamma}}$ defined by the fine $k$-weighted tree $\tilde{\gamma}=(\Gamma, \tilde{w})$. Moreover, $\zeta$ is equivariant when we equip $S^{\gamma}$ and $\mathbb{A}_{X}^{d(\Gamma)}$ with the $\mathbb{G}_{a, X}$-actions corresponding to the locally nilpotent A-derivations $\partial_{a, m} \in \operatorname{Der}_{A}\left(B^{\gamma}\right)$ (see 2.3) and $\tilde{\partial}_{\tilde{\gamma}, a, m} \in \operatorname{Der}_{A}(A[\Gamma])$ (Proposition 2.15) respectively.

Example 3.2. We consider the abstract Danielewski surface $\pi: S^{\gamma} \rightarrow X$ defined by the fine $k$-weighted tree of Example 2.4. The canonical morphism $\psi: S^{\gamma} \rightarrow$ $\mathbb{A}_{X}^{1}=\operatorname{Spec}\left(k[x]\left[X_{e_{-1}}\right]\right)$ is given by the section $s_{e_{-1}} \in B^{\gamma}$ whose restrictions on the canonical open subsets $S_{i}=\operatorname{Spec}\left(k[x]\left[T_{i}\right]\right)$ are given by

$$
s_{e_{-1}} \mid S_{i}= \begin{cases}(-1)^{i+1}+x T_{i}, & \text { if } i=1,2, \\ (-1)^{i+1} x+x^{2} T_{i}, & \text { if } i=3,4 .\end{cases}
$$

Letting $C_{i}=\pi^{-1}\left(x_{0}\right) \cap S_{i}, i=1, \ldots, 4$, be the irreducible components of $\pi^{-1}\left(x_{0}\right)$, we see that $s_{e_{-1}}$ restricts to a coordinate function on every fiber $\pi^{-1}(y), y \in X_{*}$, and is locally constant on $\pi^{-1}\left(x_{0}\right)$ with the values $1,-1$ and 0 on $C_{1}, C_{2}$ and $C_{3} \cup C_{4}$ respectively. Therefore, letting $P(t)=\left(t^{2}-1\right) \in k[t]$, the section $x^{-1} s_{e_{-1}} P\left(s_{e_{-1}}\right) \in$ $B^{\gamma} \otimes_{k[x]} k\left[x, x^{-1}\right]$ extends to a section $s_{e_{0}} \in B^{\gamma}$ whose restrictions on the $S_{i}$ 's are given by

$$
\left.s_{e_{0}}\right|_{i}= \begin{cases}2 T_{1}+3 x T_{1}^{2}+x^{2} T_{1}^{3}, & \text { if } i=1, \\ 2 T_{2}-3 x T_{2}^{2}+x^{2} T_{2}^{3}, & \text { if } i=2, \\ -1-x T_{3}+x^{2} \xi_{3}\left(x, T_{3}\right), & \text { if } i=3, \\ 1-x T_{4}+x^{2} \xi_{4}\left(x, T_{4}\right), & \text { if } i=4,\end{cases}
$$

for certain polynomials $\xi_{3}(x, t), \xi_{4}(x, t) \in k[x, t]$. Thus $s_{e_{0}}$ restricts to a coordinate function on $C_{1}$ and $C_{2}$, and is constant on $C_{3}$ and $C_{4}$ with the values -1 and 1 respectively. Again, $x^{-1} P\left(s_{e_{0}}\right) \in B_{\gamma} \otimes_{k[x]} k\left[x, x^{-1}\right]$ extends to a regular function on $S_{3} \cup S_{4} \subset S^{\gamma}$ which restricts to a coordinate function on $C_{3}$ and $C_{4}$. Clearly, $x^{-1} P\left(s_{e_{-1}}\right) P\left(s_{e_{0}}\right)$ extends to a section $s_{e_{1}} \in B^{\gamma}$ with the same property as 
$\left.P\left(s_{e_{-1}}\right)\right|_{C_{i}}=-1, i=3,4$. The $A$-algebra homomorphism $A\left[X_{e_{-1}}, X_{e_{0}}, X_{e_{1}}\right] \rightarrow$ $B^{\gamma}, X_{e} \mapsto s_{e}$ defines a closed embedding $\zeta: S^{\gamma} \rightarrow \mathbb{A}_{X}^{3}$, inducing an $X$-isomorphism between $S^{\gamma}$ and the embedded Danielewski surface $S_{\tilde{\gamma}}$ defined by the fine $k$-weighted tree $\tilde{\gamma}=(\Gamma, \tilde{w})$ of Example 2.16.

3.3. To prove Theorem 3.1 , we proceed in a similar way as in the previous example. More precisely, given an abstract Danielewski surface $S^{\gamma}$ defined by a fine $k$-weighted tree $\gamma=(\Gamma, w)$, we construct in 3.4 and Lemmas 3.5-3.7 below a fine weight function $\tilde{w}: E(\Gamma) \rightarrow k$ on $\Gamma$ and a collection of sections $s_{e} \in B^{\gamma}, e \in \mathbf{P}(\Gamma) \cup\left\{e_{-1}\right\}$, which define a closed embedding $\zeta: S^{\gamma} \hookrightarrow \mathbb{A}_{X}^{d(\Gamma)}$ inducing an $X$-isomorphism $\phi: S^{\gamma} \stackrel{\sim}{\rightarrow}$ $S_{\tilde{\gamma}}$ between $S^{\gamma}$ and the embedded Danielewski surface defined by the tree $\tilde{\gamma}=(\Gamma, \tilde{w})$.

3.4. Given a fine $k$-weighted tree $\gamma=(\Gamma, w)$ with the leaves $e_{1, m_{1}}, \ldots, e_{n, m_{n}}$, we denote by $\tau_{i}: B^{\gamma}=\Gamma\left(S^{\gamma}, \mathcal{O}_{S^{\gamma}}\right) \rightarrow A\left[T_{i}\right]$ the localization homomorphisms corresponding to the canonical open covering of the abstract Danielewski surface $S^{\gamma}$ by the open subsets $S_{i}=\operatorname{Spec}\left(A\left[T_{i}\right]\right), i=1, \ldots, n$. The canonical $X$-morphism $\psi: S^{\gamma} \rightarrow \mathbb{A}_{X}^{1}=\operatorname{Spec}\left(A\left[X_{e_{-1}}\right]\right)(2.2)$ corresponds to the section $s_{e_{-1}} \in B^{\gamma}$ such that

$$
\tau_{i}\left(s_{e_{-1}}\right)=\sum_{j=0}^{m_{i}} w_{i, j} x^{j} \in A\left[T_{i}\right],
$$

where

$$
w_{i, j}= \begin{cases}w\left(\overleftarrow{e_{i, j} e_{i, j+1}}\right), & \text { if } 0 \leq j \leq m_{i}-1, \\ T_{i}, & \text { if } j=m_{i}\end{cases}
$$

For every $e \in \Gamma$, we let

$$
C_{e}=\bigsqcup_{\left\{e_{i, m_{i}} \in L\left((\uparrow e)_{\Gamma}\right)\right\}}\left(\pi^{-1}\left(x_{0}\right) \cap S_{i}\right) \simeq \operatorname{Spec}\left(\prod_{\left\{e_{i, m_{i}} \in L\left((\uparrow e)_{\Gamma}\right)\right\}} \operatorname{Spec}\left(k\left[T_{i}\right]\right)\right) .
$$

If $\gamma$ has height $h=0$ then $\Gamma$ is the trivial tree with one element $\left\{e_{0}\right\}$ and $\psi: S^{\gamma} \rightarrow \mathbb{A}_{X}^{1}$ is an isomorphism. Otherwise, if $h \geq 1$, then we have the following result.

Lemma 3.5. If $h \geq 1$ then there exists a fine weight function $\tilde{w}: E(\Gamma) \rightarrow k$, $\overleftarrow{e^{\prime} e} \mapsto \tilde{a}_{e^{\prime}, e}$ defining a fine $k$-weighted tree $\tilde{\gamma}=(\Gamma, \tilde{w})$, and a collection of sections $\left(s_{e}\right)_{e \in \mathbf{P}(\Gamma) \cup\left\{e_{-1}\right\}} \in B^{\gamma}$ with the following properties.

a) For every $e_{i, j} \in \mathbf{P}(\Gamma), s_{e_{i, j}}=x^{-1} G_{e_{i, j}}(\tilde{\gamma})\left(s_{e_{-1}}, s_{e_{0}}, s_{e_{i, 1}}, \ldots, s_{e_{i, j-1}}\right)$.

b) If $\mathrm{Ch}\left(e_{i, j}\right)=\left\{e_{i_{1}, j+1}, \ldots, e_{i_{r}, j+1}\right\}$, then $s_{e_{i, j-1}}$ is constant on $C_{e_{i_{l}, j+1}} \subset \pi^{-1}\left(x_{0}\right)$ with the value $\tilde{a}_{e_{i, j}, e_{i, j+1}} \in k, l=1, \ldots, r$.

c) For every leaf $e_{i, m_{i}}$ of $\Gamma, s_{e_{i, m_{i}-1}}$ induces an coordinate function on $C_{e_{i, m_{i}}} \simeq \mathbb{A}_{k}^{1}$. 
Proof. We construct the weight function $\tilde{w}$ and the sections $s_{e}$ by induction as follows. For every $m=0, \ldots, h$, we denote by $\Gamma_{m}$ the subtree of $\Gamma$ with the elements $e \in \Gamma$ at levels $l \leq m$. At step $m$, we suppose that the weight function $\tilde{w}_{m}: E\left(\Gamma_{m}\right) \rightarrow$ $k$ is constructed on $\Gamma_{m}$, as well as the sections $s_{e}$ for every $e \in \Gamma_{m-2}$, and we define the sections $s_{e}, e \in \Gamma_{m-1} \backslash \Gamma_{m-2}$. Then we extend $\tilde{w}_{m}$ to a weight function $\tilde{w}_{m+1}: E\left(\Gamma_{m+1}\right) \rightarrow k$.

Step 0 . We let $s_{e_{-1}} \in B^{\gamma}$ be the section corresponding to the canonical morphism $\psi: S^{\gamma} \rightarrow \mathbb{A}_{X}^{1}$. By definition, $\tau_{i}\left(s_{e_{-1}}\right)=w_{i, 0}+x \xi_{i}$ for a certain $\xi_{i} \in A\left[T_{i}\right]$ for every $i=1, \ldots, n$. Thus $\mathrm{b}$ ) is satisfied provided that we define the weight function $\tilde{w}_{1}$ on $\Gamma_{1} \backslash\left\{e_{0}\right\}$ by

$$
\tilde{a}_{e_{0}, e_{i, 1}}=\tilde{w}_{1}\left(\overleftarrow{e_{0} e_{i, 1}}\right)=\left.s_{e_{-1}}\right|_{C_{e_{i, 1}}}=w_{i, 0} \in k
$$

for every $e_{i, 1} \in \mathrm{Ch}\left(e_{0}\right)$. Note that if $e_{j, 1}=e_{i, 1}$, then $w_{i, 0}=w_{j, 0}$ as $w_{i, 0} \neq w_{j, 0}$ if and only if $e_{0}$ is the first common ancestor of the leaves $e_{i, m_{i}}$ and $e_{j, m_{j}}$. Thus $\tilde{\gamma}_{1}=\left(\Gamma_{1}, \tilde{w}_{1}\right)$ is a fine $k$-weighted tree and we are done with Step 0.

Step 1. By construction, the rational section $x^{-1} G_{e_{0}}\left(\tilde{\gamma}_{1}\right)\left(s_{\gamma, e_{-1}}\right) \in B^{\gamma} \otimes_{A} A_{x}$ extends to a section $s_{e_{0}}$ of $B^{\gamma}$ satisfying a). Since $\gamma$ is a fine $k$-weighted tree, we deduce from Taylor's Formula that for every $i=1, \ldots, n$, there exists a pair $\left(\alpha_{i, 1}=F_{e_{0}}^{\left\{e_{i, 1}\right\}}\left(w_{i, 0}\right), \beta_{i, 1}\right) \in k^{*} \times k$ depending only of the subchain $\left(\downarrow e_{i, 1}\right)_{\Gamma}$, and a polynomial $\xi_{i, 1} \in A\left[T_{i}\right]$ such that

$$
\tau_{i}\left(s_{e_{0}}\right)=\alpha_{i, 1} w_{i, 1}+\beta_{i, 1}+x \xi_{i, 1} \in A\left[T_{i}\right] .
$$

Thus, if $e_{i, 1}$ is a leaf of $\Gamma$ then $w_{i, 1}=T_{i}$ and so c) is satisfied. Otherwise, if $e_{j, 2}$ and $e_{j^{\prime}, 2}$ are children of $e_{i, 1}$ then $\alpha_{j, 1}=\alpha_{j^{\prime}, 1}=\alpha_{i, 1}$ and $\beta_{j, 1}=\beta_{j^{\prime}, 1}=\beta_{i, 1}$ as $e_{j, 1}=e_{j^{\prime}, 1}=e_{i, 1}$, whereas $w_{j, 1} \neq w_{j^{\prime}, 1}$ as $\gamma$ is a fine $k$-weighted tree. Thus $\tilde{\gamma}_{2}=\left(\Gamma_{2}, \tilde{w}_{2}\right)$ is a fine $k$-weighted tree for the weight function $\tilde{w}_{2}: E\left(\Gamma_{2}\right) \rightarrow k$ restricting to $\tilde{w}_{1}$ on $\Gamma_{1} \subset \Gamma_{2}$ and such that

$$
\tilde{a}_{e_{i, 1}, e_{i, 2}}=\tilde{w}_{2}\left(\overleftarrow{e_{i, 1} e_{i, 2}}\right)=\left.s_{e_{0}}\right|_{e_{e_{i, 2}}}=\left(\alpha_{i, 0} w_{i, 1}+\beta_{i, 1}\right) \in k, \quad i=1, \ldots, n
$$

By construction, b) is also satisfied. This completes Step 1.

Step $m, m \geq 2$. By induction hypothesis, $\tilde{\gamma}_{m}=\left(\Gamma_{m}, \tilde{w}_{m}\right)$ is a fine $k$-weighted tree, and the sections $s_{e} \in B^{\gamma}, e \in \Gamma_{m-2}$, satisfying the hypothesis of Lemma 3.5 have been defined. So the formula

$$
s_{e_{i, m-1}}=x^{-1} G_{e_{i, m-1}}\left(\tilde{\gamma}_{m}\right)\left(s_{e_{-1}}, s_{e_{0}}, s_{e_{i, 1}}, \ldots, s_{e_{i, m-2}}\right)
$$

makes sense and defines an element of $B^{\gamma} \otimes_{A} A_{x}$. Similarly as in Step 1, we deduce from Taylor's Formula that for every $j=0, \ldots, m-1$ there exists a pair $\left(\tilde{\alpha}_{i, j}, \tilde{\beta}_{i j}\right) \in k^{*} \times k$ depending only on the subchain $\left(\downarrow e_{i, j}\right)_{\Gamma}$, and a polynomial $\tilde{\xi}_{i, j} \in A\left[T_{i}\right]$ such that

$$
\tau_{i}\left(s_{e_{i, j-1}}\right)=a_{e_{i, j+1} e_{i, j}}+x\left(\tilde{\alpha}_{i, j} w_{i, j+1}+\tilde{\beta}_{i, j}\right)+x^{2} \tilde{\xi}_{i, j} \in A\left[T_{i}\right] .
$$


By applying Taylor's Formula again, we conclude that there exists a pair $\left(\alpha_{i, m}, \beta_{i, m}\right) \in$ $k^{*} \times k$ depending only on the subchain $\left(\downarrow e_{i, m}\right)_{\Gamma}$ and a polynomial $\xi_{i, m} \in A\left[T_{i}\right]$ such that

$$
\tau_{i}\left(s_{e_{i, m-1}}\right)=\alpha_{i, m} w_{i, m}+\beta_{i, m}+x \xi_{i, m} \in A\left[T_{i}\right] .
$$

Thus, if $e_{i, m-1} \in\left(\downarrow e_{j, m_{j}}\right)$ then $e_{i, m-1}=e_{j, m-1}$ and so $\tau_{j}\left(s_{e_{i, m-1}}\right) \in A\left[T_{j}\right]$. Otherwise, for every index $j$ such that $e_{i, m-1} \notin\left(\downarrow e_{j, m_{j}}\right)_{\Gamma}$, the first common ancestor of $e_{i, m-1}$ and $e_{j, m_{j}}$ is an element $e_{i, l}=e_{j, l}$ at level $l \leq \min \left(m-2, m_{j}-1\right)$. Thus $\left(X_{e_{j, l-1}}-\tilde{a}_{e_{j, l}, e_{j, l+1}}\right)$ divides the genealogical polynomial $G_{e_{i, m-1}}\left(\tilde{\gamma}_{m}\right)$ of $e_{i, m-1}$. Since $\tau_{j}\left(s_{e_{j, l-1}}-\tilde{a}_{e_{j, l}, e_{j, l+1}}\right) \in x A\left[T_{j}\right]$, we conclude that

$$
x \tau_{j}\left(s_{e_{i, m-1}}\right)=G_{e_{i, m-1}}\left(\tilde{\gamma}_{m}\right)\left(\tau_{j}\left(s_{e_{-1}}\right), \tau_{j}\left(s_{e_{i, 0}}\right), \tau_{j}\left(s_{e_{i, 1}}\right), \ldots, \tau_{j}\left(s_{e_{i, m-2}}\right)\right) \in x A\left[T_{j}\right] .
$$

Thus $\tau_{j}\left(s_{e_{i, m-1}}\right) \in A\left[T_{j}\right]$ for every $j=1, \ldots n$, and hence, $s_{\gamma, e_{i, m-1}} \in B^{\gamma}$. If $e_{i, m}$ is a leaf of $\Gamma$ then $w_{i, m}=w_{i, m_{i}}=T_{i}$ by definition. Thus $s_{e_{i, m-1}}$ satisfies a) and c). Finally, the same argument as in Step 1 shows that $\tilde{\gamma}_{m+1}=\left(\Gamma_{m+1}, \tilde{w}_{m+1}\right)$ is a fine $k$-weighted tree for the weight function $\tilde{w}_{m+1}: E\left(\Gamma_{m+1}\right) \rightarrow k$ restricting to $\tilde{w}_{m}$ on $\Gamma_{m} \subset \Gamma_{m+1}$ and such that

$$
\tilde{a}_{e_{i, m}, e_{i, m+1}}=\tilde{w}_{m+1}\left(\overleftarrow{e_{i, m} e_{i, m+1}}\right)=\left.s_{e_{i, m-1}}\right|_{C\left(e_{i, m+1}\right)}=\left(\alpha_{i, m} w_{i, m}+\beta_{i, m}\right) \in k,
$$

whenever $e_{i, m}$ is not a leaf of $\Gamma$. This completes Step $m$ as b) is satisfied by construction.

After $h=h(\Gamma)$ steps, the above procedure stops, and we obtain a fine $k$-weighted tree $\tilde{\gamma}=\tilde{\gamma}_{h}=\left(\Gamma, \tilde{w}_{h}\right)$ and a collection of sections $\left(s_{e}\right)_{e \in \mathbf{P}(\Gamma) \cup\left\{e_{-1}\right\}} \in B^{\gamma}$ satisfying conditions a), b) and c). This completes the proof.

The following lemma implies the first assertion of Theorem 3.1.

Lemma 3.6. The $X$-morphism $\zeta: S^{\gamma} \rightarrow \mathbb{A}_{X}^{d(\Gamma)}$ induced by the A-algebra homomorphism $\zeta^{*}: A[\Gamma] \rightarrow B^{\gamma}, X_{e} \mapsto s_{e}, e \in \mathbf{P}(\Gamma) \cup\left\{e_{-1}\right\}$, is a closed embedding inducing an $X$-isomorphism $\phi: S^{\gamma} \stackrel{\sim}{\rightarrow} S_{\tilde{\gamma}}$.

Proof. By construction, $s_{e_{-1}}$ corresponds to the canonical birational morphism $\psi: S^{\gamma} \rightarrow \mathbb{A}_{X}^{1}$, whence induces a $X_{*}$-isomorphism $\left.S^{\gamma}\right|_{X_{*}} \stackrel{\sim}{\rightarrow} \mathbb{A}_{X_{*}}^{1}$. By b) of Lemma 3.5, for every pair $e_{i, m_{i}}, e_{j, m_{j}}$ of leaves of $\Gamma$ with first common ancestor $e \in \Gamma$, the section $s_{\operatorname{Par}(e)}$ takes distinct constant values on $C_{e_{i, m_{i}}}$ and $C_{e_{i, m_{j}}}$. Thus $\zeta$ distinguishes the irreducible components of the fiber $\pi^{-1}\left(x_{0}\right)$. Finally, c) of Lemma $3.5 \mathrm{im}$ plies that for every $i=1, \ldots, n, s_{e_{i, m_{i}-1}}$ induces a coordinate function on $C_{e_{i, m_{i}}} \simeq \mathbb{A}_{k}^{1}$. This proves that $\zeta: S^{\gamma} \rightarrow \mathbb{A}_{X}^{d(\Gamma)}$ is an embedding. By construction, $\zeta^{*}\left(\Delta_{e}(\tilde{\gamma})\right)=0$ in $B^{\gamma}$ for every $e \in \mathbf{P}(\Gamma)$. Thus $x \zeta^{*}\left(\Delta_{e^{\prime}, e}(\tilde{\gamma})\right)=\zeta^{*}\left(x \Delta_{e^{\prime}, e}(\tilde{\gamma})\right)=0$ for every $\left(e, e^{\prime}\right) \in\left(\mathbf{P}(\Gamma) \backslash\left\{e_{0}\right\}\right) \times\left((\downarrow e)_{\Gamma} \backslash\left\{e_{0}\right\}\right)$ by virtue of $(2.2)$, and so, $\zeta^{*}\left(\Delta_{e^{\prime}, e}(\tilde{\gamma})\right)=0$ 
as $B^{\gamma}$ is an integral $A$-algebra. This proves that the image of $\zeta$ in contained in the embedded Danielewski surface $S_{\tilde{\gamma}}$. It is clear by construction that the induced $X$ morphism $\phi: S^{\gamma} \rightarrow S_{\tilde{\gamma}}$ restricts to a bijection between the sets of closed points of $S^{\gamma}$ and $S_{\tilde{\gamma}}$ respectively. So the result follows from Zariski's Main Theorem as $S_{\tilde{\gamma}}$ is smooth over $k$, whence, in particular, normal.

The following result completes the proof of Theorem 3.1.

Lemma 3.7. For every nontrivial $\mathbb{G}_{a, X}$-action $\boldsymbol{t}_{\gamma, a, m}$ (2.3) on an abstract Danielewski surface $\pi: S^{\gamma} \rightarrow X$ defined by a fine $k$-weighted tree $\gamma=(\Gamma, w)$, the closed embedding $\zeta: S^{\gamma} \hookrightarrow \mathbb{A}_{X}^{d(\Gamma)}$ in Lemma 3.6 is equivariant when we equip $\mathbb{A}_{X}^{d(\Gamma)}$ with the $\mathbb{G}_{a, X}$-action induced by the locally nilpotent A-derivation $\tilde{\partial}_{\tilde{\gamma}, a, m} \in \operatorname{Der}_{A}(A[\Gamma])$ (Proposition 2.15).

Proof. By definition (see 2.3), the twisted translation $\boldsymbol{t}_{\gamma, a, m}$ on $S^{\gamma}$ is induced by the extension $\partial_{a, m}$ to $B^{\gamma}$ of the locally nilpotent derivation $\delta_{a, m}=a x^{m} \partial_{X_{e_{-1}}}$ of $B^{\gamma} \otimes_{A} A_{x} \simeq A_{x}\left[X_{e_{-1}}\right]$, where $m \geq h(\Gamma)$ and $a \in A \backslash\{0\}$. By construction, for every $e \in \mathbf{P}(\Gamma)$, we have $s_{e}=x^{-1} G_{e}(\tilde{\gamma})\left(s_{e_{-1}}, s_{e_{0}}, \ldots, s_{\operatorname{Par}(e)}\right) \in B^{\gamma} \subset A_{x}\left[X_{e_{-1}}\right]$ and so,

$\partial_{a, m}\left(s_{e}\right)=x^{-1} \sum_{e^{\prime} \in \operatorname{Anc}(e) \cup\left\{e_{-1}\right\}} \partial_{X_{e^{\prime}}} G_{e}(\tilde{\gamma})\left(s_{e_{-1}}, s_{e_{0}}, \ldots, s_{\operatorname{Par}(e)}\right) \partial_{a, m}\left(s_{e^{\prime}}\right) \in B^{\gamma} \otimes_{A} A_{x}$.

In view of the definition of $\tilde{\partial}_{\tilde{\gamma}, a, m} \in \operatorname{Der}_{A}(A[\Gamma])$ (see Proposition 2.15), this means precisely that the embedding $\zeta: S^{\gamma} \hookrightarrow \mathbb{A}_{X}^{d(\Gamma)}$ is equivariant when we equip $S^{\gamma}$ and $\mathbb{A}_{X}^{d(\Gamma)}$ with the actions corresponding to the locally nilpotent derivation $\partial_{a, m}$ and $\tilde{\partial}_{\tilde{\gamma}, a, m}$.

Corollary 3.8. Every Danielewski surface $\pi: S \rightarrow X$ equipped with a nontrivial $\mathbb{G}_{a, X}$-action is equivariantly $X$-isomorphic to an embedded Danielewski surface $S_{\gamma}$ defined by a fine $k$-weighted tree $\gamma=(\Gamma, w)$, equipped with the $\mathbb{G}_{a, X}$-action corresponding to a suitable locally nilpotent derivation $\partial_{\gamma, a, m} \in \operatorname{Der}_{A}\left(B_{\gamma}\right)$, where $m \geq h(\Gamma)$ and $a \in A \backslash\{0\}$.

Proof. By Theorem 3.2 in [4], every Danielewski surface $S$ is isomorphic to an abstract Danielewski surface $S^{\gamma}$ defined by a fine $k$-weighted tree $\gamma$. Moreover, by Proposition 2.12 in loc. cit., every nontrivial $\mathbb{G}_{a, X}$-action on $S^{\gamma}$ coincides with a twisted translation $\boldsymbol{t}_{\gamma, a, m}$ for a suitable pair $(m \geq h(\Gamma), a \in A \backslash\{0\})$. So the result follows from Theorem 3.1.

Corollary 3.9. Every $\mathbb{G}_{a, X}$-action on an embedded Danielewski surface $S_{\gamma}$ defined by a fine $k$-weighted tree $\gamma=(\Gamma, w)$ is induced by a locally nilpotent derivation $\partial_{\gamma, a, m} \in \operatorname{Der}_{A}\left(B_{\gamma}\right)$. 
Since the locally nilpotent derivations $\partial_{\gamma, a, m} \in \operatorname{Der}_{A}\left(B_{\gamma}\right)$ are induced by locally nilpotent derivations $\tilde{\partial}_{\gamma, a, m} \in \operatorname{Der}_{A}(A[\Gamma])$, we obtain the following result.

Corollary 3.10. Every Danielewski surface $\pi: S \rightarrow X$ admits a closed embedding $\zeta: S \hookrightarrow \mathbb{A}_{X}^{d}$ into a relative affine space $\mathbb{A}_{X}^{d}$, where $d \geq 1$, such that every $\mathbb{G}_{a, X}$-action on $S$ extends to an action on $\mathbb{A}_{X}^{d}$.

In particular, if the Makar-Limanov invariant of $S$ is nontrivial, then $\pi: S \rightarrow X$ is a unique $\mathbb{A}^{1}$-fibration on $S$ up to automorphisms of $X$. Therefore, the general orbits of a $\mathbb{G}_{a, k}$-action on $S$ coincide with the general fibers of $\pi$. This leads to the following result.

Corollary 3.11. Every Danielewski surface $S$ with a nontrivial Makar-Limanov invariant admits a closed embedding into an affine space $\mathbb{A}_{k}^{d}$ in such a way that every $\mathbb{G}_{a, k}$-action on $S$ extends to an action on $\mathbb{A}_{k}^{d}$.

Morphisms of Danielewski surfaces as linear projections. A morphism of Danielewski surfaces is a birational $X$-morphism $\beta: S^{\prime} \rightarrow S$, restricting to an isomorphism over $X_{*}$. In other words, $\beta$ is an affine modification [7] restricting to an isomorphism over the complement of the support of the principal divisor $\pi^{-1}\left(x_{0}\right)=\operatorname{div}(x) \subset S$. Thus, letting $S=\operatorname{Spec}(B)$, there exists an ideal $I \subset B$ containing a power $x^{m}$ of $x$ such that $S^{\prime}$ is isomorphic to the open subset $\operatorname{Spec}\left(B[I t] /\left(1-x^{m} t\right)\right)$ of the spectrum of the Rees algebra $B[I t]$. In turn, this implies that $S^{\prime} \simeq \operatorname{Spec}\left(B\left[t_{1}, \ldots, t_{r}\right] / J\right)$ for a certain ideal $J$. In these coordinates, the morphism $\beta: S^{\prime} \rightarrow S$ coincides with the restriction to $S^{\prime}$ of the projection $\operatorname{pr}_{S}: \mathbb{A}_{S}^{r+1}=\operatorname{Spec}\left(B\left[t_{1}, \ldots, t_{r}\right]\right) \rightarrow S$. Here we give a more precise description of this situation.

3.12. To every morphism $\tau: \gamma^{\prime}=\left(\Gamma^{\prime}, w^{\prime}\right) \rightarrow \gamma=(\Gamma, w)$ of fine $k$-weighted tree (see Definition 1.4), we associate a morphism $\beta_{\tau}: S^{\gamma^{\prime}} \rightarrow S^{\gamma}$ between the associated abstract Danielewski surfaces in the following manner. We let $\sigma^{\prime}=\left\{\sigma_{i}^{\prime} \in A\right\}_{i=1, \ldots, n^{\prime}}$ and $\sigma=\left\{\sigma_{j} \in A\right\}_{j=1, \ldots, n}$ be the collection of polynomials associated with $\gamma^{\prime}$ and $\gamma$, and we let $g^{\prime}=\left\{g_{i j}^{\prime} \in A_{x}\right\}$ and $g=\left\{g_{i j} \in A_{x}\right\}$ be the corresponding transition functions. We denote by $S_{i}^{\prime}=\operatorname{Spec}\left(A\left[T_{i}^{\prime}\right]\right), i=1, \ldots, n^{\prime}$, and $S_{j}=\operatorname{Spec}\left(A\left[T_{j}\right]\right)$, $j=1, \ldots, n$, the open subsets of the canonical coverings of $S^{\gamma^{\prime}}$ and $S^{\gamma}$ respectively. By Remark 1.5, the image of a leaf $e_{i, m_{i}^{\prime}}^{\prime}$ of $\Gamma^{\prime}$ by $\tau$ is a leaf $e_{j(i), m_{j(i)}}$ of $\Gamma$ such that $m_{i}^{\prime} \geq m_{j(i)}$ and $\tau\left(e_{i, k}^{\prime}\right)=e_{j(i), \min \left(k, m_{j(i)}\right)}$ for every $k=0, \ldots, m_{i}^{\prime}$. Since $w\left(\overleftarrow{\tau\left(e_{i, k}^{\prime}\right) \tau\left(e_{i, k+1}^{\prime}\right)}\right)=w^{\prime}\left(\overleftarrow{e_{i, k}^{\prime} e_{i, k+1}^{\prime}}\right)$ whenever $\tau\left(e_{i, k}^{\prime}\right) \neq \tau\left(e_{i, k+1}^{\prime}\right)$, we conclude that there exists a collection $\sigma^{\prime \prime}=\left\{\sigma_{i}^{\prime \prime} \in A\right\}_{i=1, \ldots, n^{\prime}}$ such that $\sigma_{i}^{\prime}=\sigma_{j(i)}+x^{m_{j(i)}} \sigma_{i}^{\prime \prime} \in A$ for every $i=1, \ldots, n^{\prime}$. Then for every $i=1, \ldots, n^{\prime}$, the $A$-algebra homomorphism

$$
A\left[T_{j(i)}\right] \longrightarrow A\left[T_{i}^{\prime}\right], \quad T_{j(i)} \mapsto \sigma_{i}^{\prime \prime}+x^{m_{i}^{\prime}-m_{j(i)}} T_{i}^{\prime}
$$


defines a birational $X$-morphism $\beta_{\tau}^{(i)}: S_{i}^{\prime} \rightarrow S_{j(i)}$ restricting to an isomorphism over $X_{*}$. Since the transition functions satisfy the relation $x^{m_{i^{\prime}}-m_{j(i)}} g_{i l}^{\prime}=g_{j(i) j(l)}+$ $x^{m_{j(l)}-m_{j(i)}} \sigma_{l}^{\prime \prime}+\sigma_{i}^{\prime \prime}$ for every $i, l=1, \ldots, n^{\prime}$, it follows that these local morphisms $\beta_{\tau}^{(i)}$ glue to a morphism of Danielewski surfaces $\beta_{\tau}: S^{\gamma^{\prime}} \rightarrow S^{\gamma}$. By Proposition 3.8 and Corollary 3.9 in [4], for every morphism of Danielewski surfaces $\beta: S^{\prime} \rightarrow$ $S$, there exists $X$-isomorphisms $\phi^{\prime}: S^{\prime} \stackrel{\sim}{\rightarrow} S^{\gamma^{\prime}}$ and $\phi: S \stackrel{\sim}{\rightarrow} S^{\gamma}$ for suitable fine $k$-weighted trees $\gamma^{\prime}$ and $\gamma$ such that $\phi \circ \beta \circ\left(\phi^{\prime}\right)^{-1}$ is the morphism $\beta_{\tau}$ induced by a morphism of fine $k$-weighted tree $\tau: \gamma^{\prime} \rightarrow \gamma$.

3.13. Every morphism of fine $k$-weighted tree $\tau: \gamma^{\prime} \rightarrow \gamma$ factors through a surjective morphism $\tau^{\prime}: \gamma^{\prime} \rightarrow \tau\left(\gamma^{\prime}\right)$ followed by an injection $\tau\left(\gamma^{\prime}\right) \hookrightarrow \gamma$. As a consequence, every morphism of Danielewski surfaces factors through a quasi-surjective morphism $\beta^{\prime}: S^{\gamma^{\prime}} \rightarrow S^{\tau\left(\gamma^{\prime}\right)}$, i.e. a morphism of Danielewski surfaces such that $\beta^{\prime-1}(C) \neq \varnothing$ for every irreducible component $C$ of the fiber $\pi_{\tau\left(\gamma^{\prime}\right)}^{-1}\left(x_{0}\right) \subset S^{\tau\left(\gamma^{\prime}\right)}$ followed by the open immersion of $S^{\tau\left(\gamma^{\prime}\right)}$ in $S^{\gamma}$ as the complement of irreducible components of $\pi_{\gamma}^{-1}\left(x_{0}\right) \subset S^{\gamma}$ corresponding to the leaves of $\Gamma$ which are not in the image of $\tau$.

3.14. Given a fine $k$-weighted tree $\gamma=(\Gamma, w)$, we consider the tree $\tilde{\gamma}=(\Gamma, \tilde{w})$ constructed in Lemma 3.5. For every edge $\overleftarrow{e^{\prime} e}$ of $\Gamma$, the weight $\tilde{w}\left(\overleftarrow{e^{\prime} e}\right) \in k$ is uniquely determined by the weights $w$ of the edges of the subtree of $\Gamma$ with elements $(\downarrow e)_{\Gamma} \cup \bigcup_{e^{\prime} \in(\downarrow e)_{\Gamma}} \mathrm{Ch}\left(e^{\prime}\right)$. Therefore, every surjective morphism of fine $k$-weighted trees $\tau: \gamma^{\prime}=\left(\Gamma^{\prime}, w^{\prime}\right) \rightarrow \gamma$ gives rise to a surjective morphism of fine $k$-weighted trees $\tilde{\tau}: \tilde{\gamma}^{\prime}=\left(\Gamma^{\prime}, \tilde{w}^{\prime}\right) \rightarrow \tilde{\gamma}$ which restricts to the same morphism as $\tau$ between the underlying trees $\Gamma^{\prime}$ and $\Gamma$ of $\tilde{\gamma}^{\prime}$ and $\tilde{\gamma}$ respectively ${ }^{1}$. Since the subset $\Gamma^{\prime \prime}=\left\{e^{\prime} \in\right.$ $\left.\Gamma^{\prime}, \tau^{-1}\left(\tau\left(e^{\prime}\right)\right)=\left\{e^{\prime}\right\}\right\} \subset \Gamma^{\prime}$ is a subtree of $\Gamma^{\prime}$ isomorphic to $\Gamma$, we obtain that

$$
A\left[\Gamma^{\prime}\right]=A\left[\Gamma^{\prime \prime}\right] \otimes_{A} A\left[\left(X_{e^{\prime}}\right)_{e^{\prime} \in \mathbf{P}\left(\Gamma^{\prime}\right) \cap\left(\Gamma^{\prime} \backslash \mathbf{P}\left(\Gamma^{\prime \prime}\right)\right)} \simeq A[\Gamma] \otimes_{A} A\left[\left(X_{e^{\prime}}\right)_{e^{\prime} \in \mathbf{P}\left(\Gamma^{\prime}\right) \cap\left(\Gamma^{\prime} \backslash \mathbf{P}\left(\Gamma^{\prime \prime}\right)\right)}\right] .\right.
$$

Moreover, for every $e^{\prime} \in \mathbf{P}\left(\Gamma^{\prime \prime}\right)$, the genealogical polynomial $G_{e^{\prime}}\left(\tilde{\gamma^{\prime}}\right)$ of $e^{\prime}$ is an element of $A\left[\Gamma^{\prime \prime}\right] \subset A\left[\Gamma^{\prime}\right]$ which coincides with the genealogical polynomial $G_{\tau\left(e^{\prime}\right)}(\tilde{\gamma}) \in A[\Gamma]$ of $\tau\left(e^{\prime}\right)$ via the isomorphism above. In turn, this implies that the genealogical matrix (see Definition 1.9) $M(\tilde{\gamma})$ of $\tilde{\gamma}$ is obtained from $M\left(\tilde{\gamma}^{\prime}\right)$ by deleting the rows corresponding to the elements in $\mathbf{P}\left(\Gamma^{\prime}\right) \backslash \mathbf{P}\left(\Gamma^{\prime \prime}\right)$. By construction of the embedding of $S^{\gamma}$ into $\mathbb{A}_{X}^{d(\Gamma)}$ as the Danielewski surface $S_{\tilde{\gamma}}$, we obtain the following result.

Theorem 3.15. Let $\tau: \gamma^{\prime}=\left(\Gamma^{\prime}, w^{\prime}\right) \rightarrow \gamma=(\Gamma, w)$ be a surjective morphism of fine $k$-weighted trees and let $\tilde{\tau}: \tilde{\gamma}^{\prime} \rightarrow \tilde{\gamma}$ be the morphism obtained above. Let

\footnotetext{
${ }^{1}$ Actually, the functor $\gamma \mapsto \tilde{\gamma}, \tau \mapsto \tilde{\tau}$ is an automorphism of the category $\mathcal{T}_{w, k}^{s}$ of fine $k$-weighted trees equipped with surjective morphisms.
} 
$\zeta^{\prime}: S^{\gamma^{\prime}} \hookrightarrow \mathbb{A}_{X}^{d\left(\Gamma^{\prime}\right)}$ and $\zeta: S^{\gamma} \hookrightarrow \mathbb{A}_{X}^{d(\Gamma)}$ are the embeddings from Lemma 3.6 of $S^{\gamma^{\prime}}$ and $S^{\gamma}$ as the Danielewski surfaces $S_{\tilde{\gamma}^{\prime}}$ and $S_{\tilde{\gamma}}$ respectively. Then $\zeta \circ \beta=p_{\Gamma^{\prime} / \Gamma} \circ \zeta^{\prime}$, where $p_{\Gamma^{\prime} / \Gamma}: \mathbb{A}_{X}^{d\left(\Gamma^{\prime}\right)} \rightarrow \mathbb{A}_{X}^{d(\Gamma)}$ is the projection induced by the inclusion $A[\Gamma] \simeq$ $A\left[\Gamma^{\prime \prime}\right] \subset A\left[\Gamma^{\prime}\right]$

\section{Danielewski surfaces with a trivial Makar-Limanov invariant}

The Makar-Limanov [6] invariant of an affine variety $V=\operatorname{Spec}(B)$ over a field $k$ of characteristic zero is the sub-algebra $\operatorname{ML}(V) \subset B$ of regular functions on $V$ which are invariant under every $\mathbb{G}_{a, k}$-action on $V$. A surface $S$ has a trivial MakarLimanov invariant $\operatorname{ML}(S)=k$ if and only if it admits two nontrivial $\mathbb{G}_{a, k}$-actions with distinct general orbits. In view of the correspondence between nontrivial $\mathbb{G}_{a, k}$ actions $\mathbb{G}_{a, k} \times S \rightarrow S$ on $S$ and quotient $\mathbb{A}^{1}$-fibrations $\pi: S \rightarrow X=S / / \mathbb{G}_{a, k}$, this means in turn that $S$ has a trivial Makar-Limanov invariant if and only if it admits two $\mathbb{A}^{1}$-fibrations with distinct general fibers. In this section, we characterize among Danielewski surfaces the ones with a trivial Makar-Limanov invariant.

\section{Danielewski surfaces defined by weighted combs}

Definition 4.1. A nontrivial (oriented) $\operatorname{comb}$ of height $h \geq 1$ is a tree $\Gamma$ such that for every $e \in \mathbf{P}(\Gamma)$ of degree $\operatorname{deg}_{\Gamma}(e) \geq 1$, all but possibly one of the children of $e$ are leaves of $\Gamma$. This means equivalently that the subtree $C_{\Gamma}=\mathbf{P}(\Gamma)=\left\{e_{0}<\cdots<\right.$ $\left.e_{h-1}\right\}$ of $\Gamma$ is a nonempty chain of length $h-1$, called the dorsal chain of $\Gamma$.

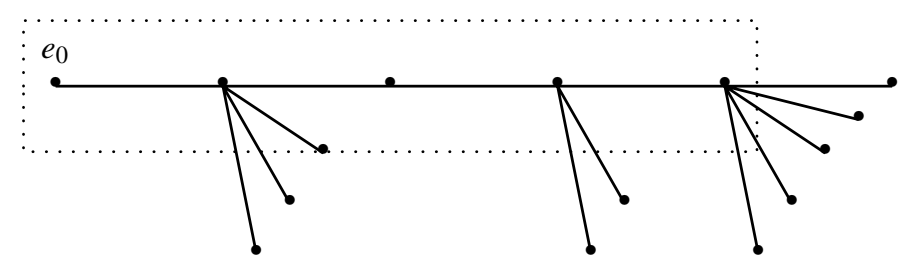

A comb rooted in $e_{0}$

4.2. By Theorem 5.4 in [4], a Danielewski surface $S$ defined over an algebraically closed field $k=\bar{k}$ of characteristic zero has a trivial Makar-Limanov invariant if and only if it is isomorphic to an abstract Danielewski surface $S^{\gamma}$ defined by a fine $k$-weighted comb. This result is based on a characterization of normal affine surfaces $S$ with a trivial Makar-Limanov invariant in terms on the boundary divisors of certain 
minimal completions $\bar{S}$ of $S$ (see [3]). Unfortunately, no such criterion exists for a normal affine surface defined over an arbitrary field $k$ of characteristic zero. However, the following result shows that the combinatorial characterization of Danielewski surfaces with a trivial Makar-Limanov invariant remains valid in this more general setting.

Theorem 4.3. A Danielewski surface $S \not \mathbb{A}_{X}^{1}$, defined over a field $k$ of characteristic zero, has a trivial Makar-Limanov invariant if and only if it is isomorphic to an abstract Danielewski surface $S^{\gamma}$ defined by a fine $k$-weighted comb. If this is the case, then there exist an integer $h \geq 1$ and a collection of monic polynomials $P_{0}, \ldots, P_{h-1} \in k[t]$ with simple roots $a_{i, j} \in k^{*}, i=0, \ldots, h-1, j=1, \ldots, \operatorname{deg}_{t}\left(P_{i}\right)$, such that $S$ is isomorphic to the surface $S_{P_{0}, \ldots, P_{h-1}} \subset \operatorname{Spec}\left(k[x]\left[y_{-1}, \ldots, y_{h-2}\right][z]\right)$ defined by the equations

$$
\begin{gathered}
x z-y_{h-2} \prod_{l=0}^{h-1} P_{l}\left(y_{l-1}\right)=0, \\
z y_{i-1}-y_{i} y_{h-2} \prod_{l=i+1}^{h-1} P_{l}\left(y_{l-1}\right)=0, \quad x y_{i}-y_{i-1} \prod_{l=0}^{i} P_{l}\left(y_{l-1}\right)=0, \quad 0 \leq i \leq h-2, \\
y_{i-1} y_{j}-y_{i} y_{j-1} \prod_{l=i+1}^{j} P_{l}\left(y_{l-1}\right)=0, \quad 0 \leq i<j \leq h-2 .
\end{gathered}
$$

4.4. The proof is given in $4.5-4.7$ below. We first observe that the condition is necessary. Indeed, suppose that the Makar-Limanov invariant of $S$ is trivial. We let $\gamma=(\Gamma, w)$ be a fine $k$-weighted tree such that $S \simeq S^{\gamma}$, and we let $i: k \hookrightarrow \bar{k}$ be the injection of $k$ in an algebraic closure $\bar{k}$. Then the Danielewski surface $S_{\bar{k}}=$ $S \times \operatorname{Spec}(k) \operatorname{Spec}(\bar{k}) \rightarrow X_{\bar{k}}=X \times_{\operatorname{Spec}(k)} \operatorname{Spec}(\bar{k})$ is $X_{\bar{k}}$-isomorphic to the abstract Danielewski surface $S^{\gamma} \times \operatorname{Spec}(k) \operatorname{Spec}(\bar{k})$ defined by the tree $\gamma$ considered a fine $\bar{k}$-weighted tree via the weight function $i \circ w: E(\Gamma) \rightarrow \bar{k}$. Since every nontrivial $\mathbb{G}_{a, k}$-action on $S$ lifts to a nontrivial action of $\mathbb{G}_{a, \bar{k}}=\mathbb{G}_{a, k} \times \operatorname{Spec}(k) \operatorname{Spec}(\bar{k})$ on $S_{\bar{k}}$, we conclude that $S_{\bar{k}}$ has a trivial Makar-Limanov invariant too. Thus the tree $\gamma$ is a comb by virtue of Theorem 5.4 in [4].

4.5. Conversely, the same argument shows that if $S$ is isomorphic to an abstract Danielewski surface $S^{\gamma}$ defined by a fine $k$-weighted comb $\gamma$, then $S_{\bar{k}}$ has a trivial Makar-Limanov invariant. Unfortunately, in general, there is no guarantee that a given $\mathbb{G}_{a, \bar{k}}$-action on $S_{\bar{k}}$ appears as the lifting of an action of $\mathbb{G}_{a, k}$ on $S$. Therefore, to show that the condition is sufficient, we must proceed in a different way. We will exploit the fact that $S$ is isomorphic to an embedded surface $S_{\gamma}$ defined by a fine $k$-weighted comb $\gamma$ to construct two explicit $\mathbb{A}^{1}$-fibrations on $S$ with distinct general fibers. 
4.6. By construction, a Danielewski surface $S$ is isomorphic to $\mathbb{A}_{X}^{1}$ if and only if it is isomorphic to an abstract surface $S^{\gamma}$ defined by a fine $k$-weighted chain $\gamma$. In this case it is also isomorphic to the surface $S_{\left\{e_{0}\right\}}$ defined by the trivial tree with one element $\left\{e_{0}\right\}$. More generally, it follows from Theorem 3.10 in [4] that every Danielewski surface $S \not \mathbb{A}_{X}^{1}$ isomorphic to an abstract Danielewski surface $S^{\gamma}$ defined by a fine $k$-weighted comb $\gamma$ is also isomorphic to a surface $S^{\gamma_{0}}$ defined by a fine $k$-weighted comb $\gamma_{0}=\left(\Gamma, w_{0}\right)$ of height $h \geq 1$, with dorsal chain $C_{\Gamma}=\left\{e_{0}<e_{1}<\cdots<e_{h-1}\right\}$, satisfying the following properties:

a) The root $e_{0}$ of $\Gamma$ as at least two children.

b) For every $i=0, \ldots, h-2, w_{0}\left(\overleftarrow{e_{i} e_{i+1}}\right)=0 \in k$.

c) There exists $e_{h} \in \mathrm{Ch}\left(e_{h-1}\right)$ such that $w_{0}\left(\overleftarrow{e_{h-1} e_{h}}\right)=0 \in k$.

By definition, the restriction of the canonical morphism $\psi: S^{\gamma_{0}} \rightarrow \mathbb{A}_{X}^{1}$ to an open subset $S_{i}=\operatorname{Spec}\left(A\left[T_{i}\right]\right)$ corresponding to a leaf $e_{i, m_{i}}$ of $\Gamma$ at level $m_{i} \geq 1$ is induced by the section $w_{0}\left(\overleftarrow{e_{m_{i}-1} e_{i, m_{i}}}\right) x^{m_{i}-1}+x^{m_{i}} T_{i}$. Thus, by applying the procedure used in the proof of Lemma 3.5 to this comb $\gamma_{0}$, we obtain a fine $k$-weighted comb $\tilde{\gamma}_{0}=\left(\Gamma, \tilde{w}_{0}\right)$ with the same underlying comb $\Gamma$ as $\gamma_{0}$ such that $\tilde{w}_{0}\left(\overleftarrow{e_{i} e_{i+1}}\right)=0 \in k$ for every $i=0, \ldots, h-1$.

4.7. By construction of the tree $\tilde{\gamma}_{0}$, there exists monic polynomials $P_{0}, \ldots, P_{h-1} \in$ $k[t]$, of degrees $\operatorname{deg}\left(P_{i}\right)=\operatorname{deg}_{\Gamma}\left(e_{i}\right)-1$, with simple roots $\tilde{a}_{e, e_{i}} \in k^{*}, e \in \mathrm{Ch}\left(e_{i}\right) \backslash$ $\left\{e_{i+1}\right\}$ respectively, such that $F_{e_{i}}\left(\tilde{\gamma}_{0}\right)=X_{e_{i-1}} P_{i}\left(X_{e_{i-1}}\right)$ for every $i=0, \ldots, h-1$. Letting $y_{-1}=X_{e_{-1}}, y_{0}=X_{e_{0}}, \ldots, y_{h-2}=X_{e_{h-2}}, z=X_{e_{h-1}}$, we conclude that the embedded Danielewski surface $S_{\tilde{\gamma_{0}}}$ is $X$-isomorphic to the surface $S_{P_{0}, \ldots, P_{h-1}}$ of Theorem 4.3. This shows that every abstract Danielewski surface $S^{\gamma} \nsucceq \mathbb{A}_{X}^{1}$ defined by a fine $k$-weighted comb $\gamma$ is $X$-isomorphic to a surface $S_{P_{0}, \ldots, P_{h-1}} \subset \mathbb{A}_{X}^{h+1}$. Thus, to complete the proof of Theorem 4.3, it suffices to show that a surface $S=$ $S_{P_{0}, \ldots, P_{h-1}}=\operatorname{Spec}(B)$ has a trivial Makar-Limanov invariant. A similar argument as in 2.10 shows that $B \otimes_{k[z]} k\left[z, z^{-1}\right] \simeq k\left[z, z^{-1}\right]\left[y_{h-2}\right]$. This means equivalently that the projection $\pi_{2}=\left.\operatorname{pr}_{z}\right|_{S}: S \rightarrow Z=\operatorname{Spec}(k[z])$ in an $\mathbb{A}^{1}$-fibration restricting to the trivial line bundle $\mathbb{A}_{Z_{*}}^{1}=\operatorname{Spec}\left(k\left[z, z^{-1}\right]\left[y_{h-2}\right]\right)$ over $Z_{*}$. Since the general fibers of the two projections $\pi_{1}=\left.\operatorname{pr}_{x}\right|_{S}: S \rightarrow X=\operatorname{Spec}(k[x])$ and $\pi_{2}: S \rightarrow Z$ do not coincide, we conclude that $S$ has a trivial Makar-Limanov invariant. This completes the proof of Theorem 4.3.

Remark 4.8. The same argument as in the proof of Proposition 2.15 applied to the fibration $\pi_{2}$ shows that the locally nilpotent derivation $z^{h} \partial_{y_{h-2}}$ of $B \otimes_{k[z]} k\left[z, z^{-1}\right] \simeq$ $k\left[z, z^{-1}\right]\left[y_{h-2}\right]$ extends to a locally nilpotent derivation of $B$, induced by a triangular $k[z]$-derivation of $k[z]\left[y_{h-2}, \ldots, y_{-1}, x\right]$. This proves that every Danielewski surface $S$ with a trivial Makar-Limanov invariant can be embedded in an affine space $\mathbb{A}_{k}^{d}$ in such a way that at least two algebraically independent $\mathbb{G}_{a, k}$-actions on $S$ extend to $\mathbb{G}_{a, k}$-actions on $\mathbb{A}_{k}^{d}$. 
Nonconjugated $\mathbb{G}_{\boldsymbol{a}}$-actions on a Danielewski surface. By a result of Daigle [2], all the $\mathbb{G}_{a, k}$-actions on a Danielewski surface $S_{P, 1}=\{x z-P(y)\}$ are conjugated under the action of the automorphism group $\operatorname{Aut}\left(S_{P, 1}\right)$ of $S_{P, 1}$.

4.9. This means that for every pair of nontrivial locally nilpotent derivations $\partial_{1}$ and $\partial_{2}$ of $B=\Gamma\left(S_{P, 1}, \mathcal{O}_{S_{P, 1}}\right)$, there exists a $k$-algebra automorphism $\phi$ of $B$ such that $\phi\left(\operatorname{Ker}\left(\partial_{1}\right)\right)=\operatorname{Ker}\left(\partial_{2}\right)$. This implies in particular that the fibers of corresponding quotient $\mathbb{A}^{1}$-fibrations $\pi_{1}: S_{P, 1} \rightarrow \mathbb{A}_{k}^{1}$ and $\pi_{2}: S_{P, 1} \rightarrow \mathbb{A}_{k}^{1}$ have the same schemetheoretic structures. By 4.7 above, a Danielewski surface $S=S_{P_{0}, \ldots, P_{h-1}}=\operatorname{Spec}(B)$ admits two $\mathbb{A}^{1}$-fibrations $\pi_{1}: S \rightarrow X=\operatorname{Spec}(k[x])$ and $\pi_{2}: S \rightarrow Z=\operatorname{Spec}(k[z])$. Moreover $\pi_{2}$ restricts to the trivial line bundle over $Z_{*}=\operatorname{Spec}\left(k\left[z, z^{-1}\right]\right)$, and a similar argument as in Lemma 2.13 shows that the fiber $\left(\pi_{2}^{-1}(0)\right)_{\text {red }}$ decomposes as a disjoint union of curves isomorphic to the affine line $\mathbb{A}_{k}^{1}$. However, we have the following result.

Lemma 4.10. If $h \geq 2$, then $\pi_{2}: S=S_{P_{0}, \ldots, P_{h-1}} \rightarrow Z$ is not a Danielewski surface over $Z$.

Proof. It suffices to show that the intersection of the fiber $\pi_{2}^{-1}(0)$ with the complement of the fiber $\pi_{1}^{-1}(0)$ is a nonreduced scheme. By (2.2), the defining ideal $I_{*}$ of $S \backslash$ $\pi_{1}^{-1}(0) \simeq \mathbb{A}_{X_{*}}^{1}$ in $k\left[x, x^{-1}\right]\left[y_{-1}, \ldots, y_{h-2}\right][z]$ is generated by the polynomials $c_{i}=$ $y_{i}-x^{-1} y_{i-1} \prod_{l=0}^{i} P_{l}\left(y_{l-1}\right), i=0, \ldots, h-2$ and $d=z-x^{-1} y_{h-2} \prod_{l=0}^{h-1} P_{l}\left(y_{l-1}\right)$. We conclude recursively that there exists a polynomial $R \in k\left[x, x^{-1}\right]\left[y_{-1}\right]$ such that

$$
d \equiv z-x^{-h} y_{-1}\left(P_{0}\left(y_{-1}\right)\right)^{h} R\left(y_{-1}\right)
$$

modulo $c_{0}, \ldots, c_{h-2}$. Since the polynomial $P_{0}$ is nonconstant (see 4.6),

$$
\begin{aligned}
\left(S \backslash \pi_{1}^{-1}(0)\right) \cap \pi_{2}^{-1}(0) & \simeq \operatorname{Spec}\left(k\left[x, x^{-1}\right]\left[y_{-1}, \ldots, y_{h-2}, z\right] /\left(I_{*}, z\right)\right) \\
& \simeq \operatorname{Spec}\left(k\left[x, x^{-1}\right]\left[y_{-1}\right] /\left(x^{-h} y_{-1}\left(P_{0}\left(y_{-1}\right)\right)^{h} R\left(y_{-1}\right)\right)\right)
\end{aligned}
$$

is clearly nonreduced whenever $h \geq 2$. This completes the proof.

4.11. The above result implies that if $h \geq 2$, then the degenerate fibers of $\pi_{1}$ and $\pi_{2}$ have different scheme-theoretic structures. Therefore two $\mathbb{G}_{a, k}$-actions on $S_{P_{0}, \ldots, P_{h-1}}$ with associated quotient $\mathbb{A}^{1}$-fibrations $\pi_{1}: S \rightarrow X$ and $\pi_{2}: S \rightarrow Z$ respectively can not be conjugated in the sense of (4.9) above. This leads to the following result.

Theorem 4.12. A Danielewski surface $S \not S_{P, 1}$ with a trivial Makar-Limanov invariant admits two algebraically independent nonconjugated $\mathbb{G}_{a, k}$-actions.

As a consequence of this description, we obtain the following characterization of ordinary Danielewski surfaces $S_{P, 1}$. 
Corollary 4.13. Let $\pi: S \rightarrow X=\operatorname{Spec}(k[x])$, where $k$ is an arbitrary field of characteristic zero, be a Danielewski surface with a trivial Makar-Limanov invariant. Then the following are equivalent.

a) $S$ admits a free $\mathbb{G}_{a, X}$-action.

b) $S$ is isomorphic to a surface $S_{P, 1}=\{x z-P(y)=0\}$ in $\mathbb{A}_{k}^{3}=\operatorname{Spec}(k[x, y, z])$, where $P$ is a nonconstant polynomial with $\operatorname{deg} P$ simple roots.

c) The canonical sheaf $\omega_{S}$ is trivial.

d) All $\mathbb{G}_{a, k}$-actions on $S$ are conjugated.

Proof. The equivalence b) $\Leftrightarrow d$ ) follows from [2] and the above discussion. All the other equivalences can be obtained in the same way as in Corollary 5.7 in [4].

\section{References}

[1] T. Bandman and L. Makar-Limanov, Affine surfaces with $A K(S)=\mathbb{C}$. Michigan J. Math. 49 (2001), 567-582. Zbl 01742561 MR 1872757

[2] D. Daigle, On locally nilpotent derivations of $k[x, y, z] /(x y-p(z))$. J. Pure Appl. Algebra 181 (2003), 181-208. Zbl 01930181 MR 1975298

[3] A. Dubouloz, Completions of normal affine surfaces with a trivial Makar-Limanov invariant. Michigan J. Math. 52 (2004), 289-308. Zbl 02112903 MR 2069802

[4] A. Dubouloz, Danielewski-Fieseler Surfaces. Transform. Groups 10 (2005), 139-162.

[5] K.H. Fieseler, On complex affine surfaces with $\mathbb{C}_{+}$-actions. Comment. Math. Helv. 69 (1994), 5-27. Zbl 0806.14033 MR 1259603

[6] S. Kaliman and L. Makar-Limanov, On the Russel-Koras contractible threefolds. J. Algebraic Geom. 6 (1997), 247-268. Zbl 0897.14010 MR 1489115

[7] S. Kaliman and M. Zaidenberg, Affine modifications and affine hypersurfaces with a very transitive automorphism group. Transform. Groups 4 (1999), 53-95. Zbl 0956.14041 MR 1669174

[8] L. Makar-Limanov, On groups of automorphisms of a class of surfaces. Israel J. Math. 69 (1990), 250-256. Zbl 0711.14022 MR 1045377

[9] L. Makar-Limanov, On the group of automorphisms of a surface $x^{n} y=p(z)$. Israel J. Math. 121 (2001), 113-123. Zbl 0980.14030 MR 1818396

Received October 26, 2004

A. Dubouloz, Institut Fourier, Laboratoire de Mathématiques, UMR5582 (UJF-CNRS), BP 74, 38402 St Martin d'Heres Cedex, France

E-mail: adrien.dubouloz@ujf-grenoble.fr 